Discrete Comput Geom 34:547-585 (2005)

DOI: $10.1007 / \mathrm{s} 00454-005-1196-9$

\title{
Apollonian Circle Packings: Geometry and Group Theory I. The Apollonian Group*
}

\author{
Ronald L. Graham, ${ }^{1}$ Jeffrey C. Lagarias, ${ }^{2}$ Colin L. Mallows, ${ }^{3}$ \\ Allan R. Wilks, ${ }^{4}$ and Catherine H. Yan ${ }^{5}$ \\ ${ }^{1}$ Department of Computer Science and Engineering, \\ University of California at San Diego, \\ La Jolla, CA 92093-0114, USA \\ graham@ucsd.edu \\ ${ }^{2}$ Department of Mathematics, University of Michigan, \\ Ann Arbor, MI 48109-1109, USA \\ lagarias@umich.edu \\ ${ }^{3}$ Avaya Labs, Basking Ridge, NJ 07920, USA \\ colinm@research.avayalabs.com \\ ${ }^{4}$ AT\&T Labs, Florham Park, NJ 07932-0971, USA \\ allan@research.att.com \\ ${ }^{5}$ Department of Mathematics, Texas A\&M University, \\ College Station, TX 77843-3368, USA \\ cyan@math.tamu.edu
}

\begin{abstract}
Apollonian circle packings arise by repeatedly filling the interstices between four mutually tangent circles with further tangent circles. We observe that there exist Apollonian packings which have strong integrality properties, in which all circles in the packing have integer curvatures and rational centers such that (curvature) $\times($ center) is an integer vector. This series of papers explain such properties.

A Descartes configuration is a set of four mutually tangent circles with disjoint interiors. An Apollonian circle packing can be described in terms of the Descartes configuration it contains. We describe the space of all ordered, oriented Descartes configurations using a coordinate system $\mathcal{M}_{\mathbb{D}}$ consisting of those $4 \times 4$ real matrices $\mathbf{W}$ with $\mathbf{W}^{T} \mathbf{Q}_{D} \mathbf{W}=\mathbf{Q}_{W}$ where $\mathbf{Q}_{D}$ is the matrix of the Descartes quadratic form $Q_{D}=x_{1}^{2}+x_{2}^{2}+x_{3}^{2}+x_{4}^{2}-\frac{1}{2}\left(x_{1}+\right.$ $\left.x_{2}+x_{3}+x_{4}\right)^{2}$ and $\mathbf{Q}_{W}$ of the quadratic form $Q_{W}=-8 x_{1} x_{2}+2 x_{3}^{2}+2 x_{4}^{2}$. On the parameter
\end{abstract}

* Ronald L. Graham was partially supported by NSF Grant CCR-0310991. Catherine H. Yan was partially supported by NSF Grants DMS-0070574, DMS-0245526 and a Sloan Fellowship. She is also affiliated with Nankai University, China. 
space $\mathcal{M}_{\mathbb{D}}$ the group $\operatorname{Aut}\left(Q_{D}\right)$ acts on the left, and $\operatorname{Aut}\left(Q_{W}\right)$ acts on the right, giving two different "geometric" actions. Both these groups are isomorphic to the Lorentz group $O(3,1)$. The right action of $\operatorname{Aut}\left(Q_{W}\right)$ (essentially) corresponds to Möbius transformations acting on the underlying Euclidean space $\mathbb{R}^{2}$ while the left action of $\operatorname{Aut}\left(Q_{D}\right)$ is defined only on the parameter space.

We observe that the Descartes configurations in each Apollonian packing form an orbit of a single Descartes configuration under a certain finitely generated discrete subgroup of Aut $\left(Q_{D}\right)$, which we call the Apollonian group. This group consists of $4 \times 4$ integer matrices, and its integrality properties lead to the integrality properties observed in some Apollonian circle packings.

We introduce two more related finitely generated groups in $\operatorname{Aut}\left(Q_{D}\right)$, the dual Apollonian group produced from the Apollonian group by a "duality" conjugation, and the superApollonian group which is the group generated by the Apollonian and dual Apollonian groups together. These groups also consist of integer $4 \times 4$ matrices. We show these groups are hyperbolic Coxeter groups.

\section{Introduction}

An Apollonian circle packing is a packing of circles arising by repeatedly filling the interstices between four mutually tangent circles with further tangent circles. We call an initial arrangement of four mutually tangent circles with distinct tangents (necessarily six of them) a Descartes configuration.

Starting from any Descartes configuration, we can recursively construct an infinite circle packing of the Euclidean plane, in which new circles are added which are tangent to three of the circles that have already been placed and have interiors disjoint from any of them. The infinite packing obtained in the limit of adding all possible such circles is called an Apollonian packing. The new circles added at each stage can be obtained using Möbius transformations of Descartes configurations in the partial packing.

An Apollonian packing is pictured in Fig. 1, in which each circle is labeled by its curvature, which is the inverse of its radius. The initial configuration consists of two circles of radius $\frac{1}{2}$ inscribed in a circle of radius 1 , the latter being assigned negative curvature -1 by a convention given in Section 3. This particular Apollonian packing has the special property that all circles in the packing have integer curvatures. We call a packing with this property integral. More remarkably, if one regards the circle centers as complex numbers, then one can place the initial circles so that every circle in the packing has "curvature $\times$ center" a Gaussian integer (element of the ring $\mathbb{Z}[i]$.) This occurs for example when the center of the outer circle is placed at $\mathbf{z}=0$ and the two circles of radius $\frac{1}{2}$ have centers at $\mathbf{z}=-\frac{1}{2}$ and at $\mathbf{z}=\frac{1}{2}$. We call a packing with this extra property strongly integral.

The object of this paper is to give a geometric explanation of the origin of these integrality properties involving both the curvatures and the circle centers. This is based on five facts:

(1) An Apollonian packing can be described in terms of the Descartes configurations it contains. 


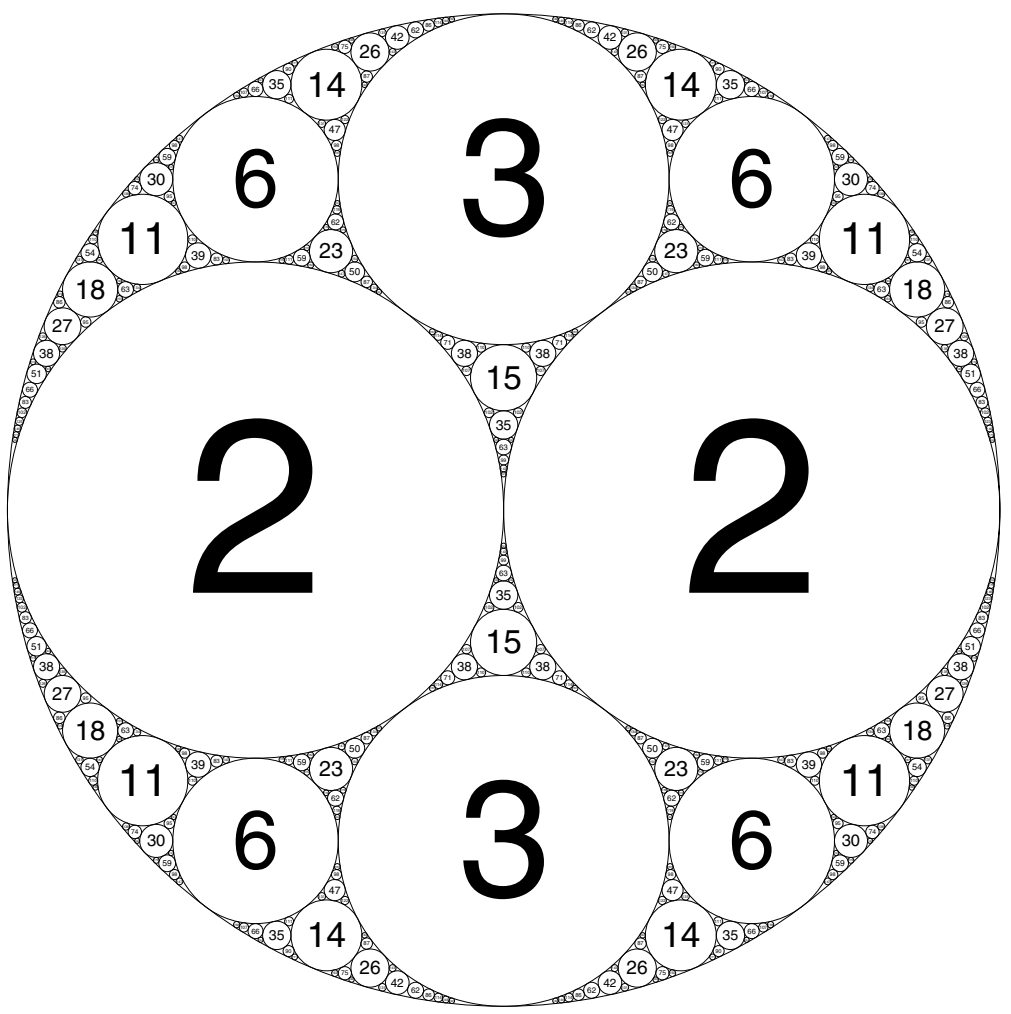

Fig. 1. An Apollonian packing, labeled with circle curvatures.

(2) There is a coordinate representation of the set $\mathcal{M}_{\mathbb{D}}$ of all ordered, oriented Descartes configurations as a six-dimensional real-algebraic variety. It consists of the set of $4 \times 4$ real matrices $\mathbf{W}$ satisfying a system of quadratic equations, $\mathbf{W}^{T} \mathbf{Q}_{D} \mathbf{W}=\mathbf{Q}_{W}$, which state that $\mathbf{W}$ conjugates the Descartes quadratic form $Q_{D}=x_{1}^{2}+x_{2}^{2}+x_{3}^{2}+x_{4}^{2}-\frac{1}{2}\left(x_{1}+x_{2}+x_{3}+x_{4}\right)^{2}$ to a quadratic form $Q_{W}=-8 x_{1} x_{2}+2 x_{3}^{2}+2 x_{4}^{2}$ that we call the Wilker quadratic form, after Wilker [42]. This coordinate system we call "augmented curvature-center coordinates," as it encodes the curvatures and centers of the circles in the configuration. We term $\mathcal{M}_{\mathbb{D}}$ with these coordinates the parameter space of ordered, oriented Descartes configurations.

(3) The variety $\mathcal{M}_{\mathbb{D}}$ is a principal homogeneous space for the Lorentz group $O(3,1)$, under both a left and a right action of this group, realized as $\operatorname{Aut}\left(Q_{D}\right)$ and $\operatorname{Aut}\left(Q_{W}\right)$, respectively. The right action corresponds to Möbius transformations acting on the plane, while the left action acts only on the Descartes configuration space.

(4) There is a discrete subgroup of the left action by $\operatorname{Aut}\left(Q_{D}\right)$, the Apollonian group $\mathcal{A}$, having the property that the (unordered, unoriented) Descartes configurations in any Apollonian packing are described by a single orbit of this group. If the Descartes configurations are regarded as ordered and oriented, then exactly 48 
such orbits correspond to each packing, each one containing a copy of each unordered, unoriented Descartes configuration in the packing.

(5) The Apollonian group $\mathcal{A}$ consists of integer $4 \times 4$ matrices.

The last property explains the existence of Apollonian packings having integrality properties of both curvatures and centers. If an initial Descartes configuration has integral augmented curvature-center coordinates, then the same property persists for all Descartes configurations in the packing, hence for all circles in the packing. The packing pictured in Fig. 1 has this property.

The observation that there are Apollonian packings having all curvatures integral is an old one, and was noted by Soddy [37], [38], who also gave an extension to three dimensions, the "bowl of integers." The existence of an integral matrix group explaining such curvatures was studied by Söderberg [39] in 1992. There are many other mentions in the literature. What we add in this direction is the observation that the circle centers can simultaneously have integrality properties.

A main contribution of these papers is the viewpoint that these properties should be understood in terms of group actions on the parameter space $\mathcal{M}_{\mathbb{D}}$ of all ordered, oriented Descartes configurations. Besides introducing coordinate systems for this parameter space (given first in [28] by three of the authors), we study the relevant discrete group actions in detail. In particular we introduce a larger group of integer matrices acting on the left, the super-Apollonian group $\mathcal{A}^{S}$, whose generators have natural geometric interpretations in terms of their action on Descartes configurations. We prove that this group is a hyperbolic Coxeter group.

The detailed contents of part I are summarized in the next section. Below we briefly indicate the contents of parts II and III.

In part II we study the integrality properties of the Apollonian packing in more detail, and their relation to the super-Apollonian group. We show that every integral Apollonian packing can be transformed to a strongly integral one by a Euclidean motion. We introduce super-packings as orbits of the super-Apollonian group, starting from a given Descartes configuration, and geometric super-packings which consist of the set of circles in all these Descartes configurations. We show that there are exactly eight different primitive ${ }^{1}$ strongly integral geometric super-packings, and that each of these contains a copy of every primitive integral Apollonian packing. We characterize the set of all (primitive) strongly integral Descartes configurations as a collection of 384 orbits of the super-Apollonian group.

In part III we consider to what extent the results proved in parts I and II extend to higher dimensions. In all dimensions $n \geq 3$ there are analogues of the parameter space of Descartes configurations, of left and right group actions by $O(n+1,1)$, and of the Apollonian, dual Apollonian and super-Apollonian groups. However, in dimensions $n \geq 4$ the Apollonian group action does not correspond to a sphere packing; the spheres will overlap. Nevertheless, one can still study the orbits of these group actions on the parameter space. The Apollonian group in higher dimensions has rational entries, rather than integer entries. We show that configurations having all curvatures rational exist in all dimensions, and having curvature $\times$ centers rational exist in certain dimensions only.

\footnotetext{
${ }^{1}$ An integral packing is primitive if the greatest common divisor of all curvatures of circles in the packing is 1 .
} 
The general framework of these papers was developed by the second author (JCL), who also did much of the writing. This paper is an extensively revised version of a preprint written in 2000, which adds some new results in Sections 4 and 5.

\section{Summary of Results}

There are at least three ways to describe the Apollonian packing $\mathcal{P}$ containing a given Descartes configuration $\mathcal{D}$ :

(G1) [Geometric] An Apollonian packing $\mathcal{P}_{\mathcal{D}}$ is a set of circles in $\hat{\mathbb{C}}=\mathbb{R}^{2} \cup\{\infty\}$, which comprise four orbits under the action of a discrete group $G_{\mathcal{A}}(\mathcal{D})$ of Möbius transformations inside the conformal group Möb(2). The discrete group $G_{\mathcal{A}}(\mathcal{D})$ depends on $\mathcal{D}$.

(G2) [Algebraic] The collection of all (ordered, oriented) Descartes configurations in the packing $\mathcal{P}_{\mathcal{D}}$ form 48 orbits of a discrete group $\mathcal{A}$, the Apollonian group, contained in the group $\operatorname{Aut}\left(\mathcal{M}_{\mathbb{D}}\right) \equiv \operatorname{Aut}\left(Q_{D}\right)$ of left-automorphisms of the parameter space $\mathcal{M}_{\mathbb{D}}$ of Descartes configurations. The discrete group $\mathcal{A}$ is independent of the configuration $\mathcal{D}$.

(G3) [Holographic] The open disks comprising the interiors of the circles in the packing are the complement $\hat{\mathbb{C}} \backslash \Lambda_{\mathcal{D}}$ of the limit set $\Lambda_{\mathcal{D}}$ of a certain Schottky group $\mathcal{S}_{\mathcal{D}}$ acting on hyperbolic 3 -space $\mathbb{H}^{3}$, with $\hat{\mathbb{C}}$ identified with its ideal boundary. The Schottky group $\mathcal{S}_{\mathcal{D}}$ depends on $\mathcal{D}$.

In this paper we mainly consider viewpoints (G1) and (G2). Viewpoint (G3) is described in Chapter 7 in [33], and we treat it in Appendix B. It is termed "holographic" because it views the limit set of the packing as the boundary of a higher-dimensional object, which in principle gives information about it. In Appendix B we point out a connection of viewpoint (G3) to viewpoint (G1). The term "algebraic" for (G2) refers to the group action being on a real-algebraic variety of Descartes configurations.

The main emphasis of this series of papers is to study Apollonian packings in terms of the Descartes configurations they contain. That is, we study the packing as a collection of points inside the parameter space of all ordered, oriented Descartes configurations. There are two different group actions on this parameter space, which are a right action associated to viewpoint (G1) and a left action corresponding to viewpoint (G2), with the groups both isomorphic to the Lorentz group $O(3,1)$, a real Lie group, as explained in Section 3. In particular, group actions are independent and mutually commute. The discrete group $G_{\mathcal{A}}(\mathcal{D})$ above is contained in the right action and the discrete group $\mathcal{A}$, the Apollonian group, is contained in the left action. Thus viewpoints (G1) and (G2) are complementary and coexist simultaneously on the space of all Descartes configurations.

As stated in the Introduction, the integer structures in the curvatures and centers of some Apollonian packings can be explained in terms of the viewpoint (G2), using the discrete group $\mathcal{A}$, the Apollonian group. This viewpoint can be traced back to the "inversive crystal" in Section 14 in [42].

In Section 3 we coordinatize the space $\mathcal{M}_{\mathbb{D}}$ of (ordered, oriented) Descartes configurations as in [28] and describe two group actions on this space. In Section 3.1 we give two coordinate systems. The first labels such a Descartes configuration with a $4 \times 3$ matrix $\mathbf{M}_{\mathcal{D}}$, called curvature-center coordinates, and the second with a $4 \times 4$ matrix $\mathbf{W}_{\mathcal{D}}$, 
called augmented curvature-center coordinates. These coordinates are characterized by quadratic relations which generalize the Descartes circle theorem. Theorem 3.1 exactly characterizes these relations for curvature-center coordinates, strengthening Theorem 3.2 in [28] by formulating and proving a converse. For the augmented curvature-center coordinates these quadratic relations (proved in [28]) take the form

$$
\mathbf{W}_{\mathcal{D}}^{T} \mathbf{Q}_{D} \mathbf{W}_{\mathcal{D}}=\mathbf{Q}_{W},
$$

which gives a conjugacy of the Descartes quadratic form $Q_{D}$ to the Wilker quadratic form $Q_{W}$. These forms are indefinite of signature $(3,1)$ and their (real) automorphism groups $\operatorname{Aut}\left(Q_{D}\right)$ and $\operatorname{Aut}\left(Q_{W}\right)$ are isomorphic to the Lorentz group $O(3,1)$.

In Section 3.2 we describe a linear left action ("Lorentz action") by $\operatorname{Aut}\left(Q_{D}\right)$ and a right action ("Möbius action") by $\operatorname{Aut}\left(Q_{W}\right)$ on the space $\mathcal{M}_{\mathbb{D}}$. Theorem 3.3 describes these actions. It shows that the space $\mathcal{M}_{\mathbb{D}}$ is a principal homogeoneous space for the group $O(3,1)$ under both the left action and the right action. The Möbius action is treated in more detail in Appendix A. In Section 3.3 we describe some integral elements of the Lorentz action $\operatorname{Aut}\left(Q_{D}\right)$ which have geometric interpretations as simple transformations of a Descartes configuration. These elements are used in defining the Apollonian group, dual Apollonian group and super-Apollonian group given below.

In Section 4 we describe Apollonian packings and the Apollonian group. Theorem 4.3 establishes the basic fact that the interiors of all circles in an Apollonian packing are disjoint. The much-studied residual set $\Lambda(\mathcal{P})$ of an Apollonian packing is the complement of the interiors of all circles; it is a set of measure zero. For later use, Theorem 5.2 gives several properties of the residual set $\Lambda(\mathcal{P})$. Theorem 4.3 shows that the (ordered, oriented) Descartes configurations in a packing form 48 orbits of this group.

In Section 5 we define the dual Apollonian group $\mathcal{A}^{\perp}$ and call its orbits dual Apollonian packings. Theorem 5.3 shows that the set of all tangency points of circles in a dual Apollonian packing has closure the limit set of another Apollonian packing, that generated by the dual Descartes configuration of any Descartes configuration generating the packing.

In Section 6 we define the super-Apollonian group $\mathcal{A}^{S}$ to be the group generated by $\mathcal{A}$ and $\mathcal{A}^{\perp}$ combined, and call its orbits Apollonian super-packings. It is a discrete group of integer matrices contained in $\operatorname{Aut}\left(Q_{D}\right)$. Theorem 6.1 in Section 6.1 gives a complete presentation for $\mathcal{A}^{\perp}$, establishing that it is a hyperbolic Coxeter group. In Section 6.2 we add remarks on super-packings, which are studied at length in part II.

In Appendix A we describe the Möbius group action in detail. Theorem A.2 gives an isomorphism of this group to $\operatorname{Aut}\left(Q_{W}\right)$.

In Appendix B we describe the Schottky group action in [33]. We indicate some relations to the Möbius group action.

\section{Descartes Configurations and Group Actions}

\subsection{Descartes Configurations and Curvature-Center Coordinates}

In 1643 Descartes found a relation between the radii for four mutually disjoint tangent circles of type (a) in Fig. 2. Let $r_{1}, r_{2}, r_{3}, r_{4}$ be the radii of the tangent circles. Descartes 


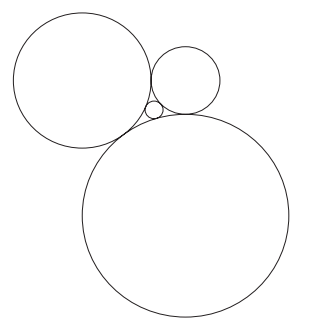

(a)

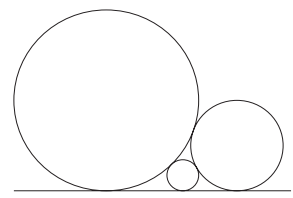

(c)

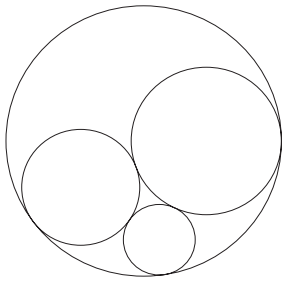

(b)

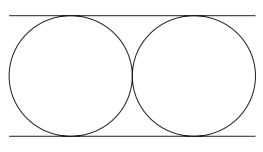

(d)

Fig. 2. Descartes configurations.

showed $^{2}$ a result equivalent to

$$
\frac{1}{r_{1}^{2}}+\frac{1}{r_{2}^{2}}+\frac{1}{r_{3}^{2}}+\frac{1}{r_{4}^{2}}=\frac{1}{2}\left(\frac{1}{r_{1}}+\frac{1}{r_{2}}+\frac{1}{r_{3}}+\frac{1}{r_{4}}\right)^{2}
$$

which is now called the Descartes circle theorem. This result can be extended to apply to all Descartes configurations, which can be of types (a)-(d) in Fig. 2.

Figure 2(a) is a standard Descartes configuration in which the circles have disjoint interiors, and all curvatures are positive. We also allow configurations like Fig. 2(b) in which one circle encloses the other three. We also allow straight lines to be regarded as circles of curvature zero, leading to Descartes configurations like Fig. 2(c) and (d), in which the "interior" of the "circle" defined by a straight line is defined to be a suitable half-plane.

To extend the Descartes circle theorem to all Descartes configurations of types (a)-(d), we must define the curvatures to have appropriate signs, as follows. An oriented circle is a circle together with an assigned direction of unit normal vector, which can point inward or outward. If it has radius $r$ then its oriented radius is $r$ for an inward pointing normal and $-r$ for an outward pointing normal. Its oriented curvature (or "signed curvature") is $1 / r$ for an inward pointing normal and $-1 / r$ for an outward pointing normal. By convention, the interior of an oriented circle is its interior for an inward pointing normal and its exterior for an outward pointing normal. An oriented Descartes configuration is a Descartes configuration in which the orientations of the circles are compatible in the following sense: either (i) the interiors of all four oriented circles are disjoint, or (ii) the interiors are disjoint when all orientations are reversed. Each Descartes configuration has exactly two compatible orientations in this sense, one obtained from the other by reversing all orientations. The positive orientation of a Descartes configuration is the

${ }^{2}$ Descartes expressed his relation in a different form, obtained by clearing denominators in (3.1). 
one in which the sum of the signed curvatures is positive, while the negative orientation is the one in which the sum of the curvatures is negative. (One can show that the sum of the signed curvatures is always nonzero.) Note that positive orientation corresponds to case (i), and negative orientation to case (ii). With these definitions, the Descartes circle theorem remains valid for all oriented Descartes configurations, using oriented curvatures.

The geometry of Descartes configurations in Fig. 2 is encoded in the curvature vector $\mathbf{b}=\left(b_{1}, b_{2}, b_{3}, b_{4}\right)^{T}$ where $b_{i}$ is the oriented curvature of the $i$ th circle. If $\sum_{j=1}^{4} b_{j}>0$, then one of the following holds: (a) all of $b_{1}, b_{2}, b_{3}, b_{4}$ are positive; (b) three are positive and one is negative; (c) three are positive and one is zero; or (d) two are positive and equal and the other two are zero.

The Descartes circle theorem gives a quadratic equation for the curvature vector $\mathbf{b}$, which can be rewritten as

$$
\mathbf{b}^{T} \mathbf{Q}_{D} \mathbf{b}=0
$$

in which

$$
\mathbf{Q}_{D}=\mathbf{I}-\frac{1}{2} \mathbf{1 1}^{T}=\frac{1}{2}\left[\begin{array}{rrrr}
1 & -1 & -1 & -1 \\
-1 & 1 & -1 & -1 \\
-1 & -1 & 1 & -1 \\
-1 & -1 & -1 & 1
\end{array}\right]
$$

is the Descartes quadratic form. Here 1 represents the vector $(1,1,1,1)^{T}$.

In [28] three of the authors of this paper showed there exist matrix extensions of the Descartes circle theorem which encode information about both the curvatures and the centers of the circles in the configuration, as follows. Given an oriented circle $C$ with center $(x, y)$ and oriented curvature $b$ we define its curvature-center coordinates to be the $1 \times 3$ row vector $\mathbf{m}(C):=(b, b x, b y)$. For the "degenerate case" of an oriented straight line $H$ we define its curvature-center coordinates as

$$
\mathbf{m}(H):=(0, \mathbf{h}),
$$

where $\mathbf{h}=\left(h_{1}, h_{2}\right)$ is the unit normal vector giving the orientation of the straight line.

Theorem 3.1 (Extended Descartes Theorem). Given an ordered configuration $\mathcal{D}$ of four oriented circles with oriented curvatures $\left(b_{1}, b_{2}, b_{3}, b_{4}\right)$ and centers $\left\{\left(x_{i}, y_{i}\right): 1 \leq\right.$ $i \leq 4\}$, let $\mathbf{M}_{\mathcal{D}}$ be the $4 \times 3$ matrix

$$
\mathbf{M}_{\mathcal{D}}:=\left[\begin{array}{lll}
b_{1} & b_{1} x_{1} & b_{1} y_{1} \\
b_{2} & b_{2} x_{2} & b_{2} y_{2} \\
b_{3} & b_{3} x_{3} & b_{3} y_{3} \\
b_{4} & b_{4} x_{4} & b_{4} y_{4}
\end{array}\right] .
$$

We include the "degenerate cases" where some circles are oriented lines. If this configuration is an oriented Descartes configuration, then $\mathbf{M}=\mathbf{M}_{\mathcal{D}}$ has a nonzero first column and satisfies

$$
\mathbf{M}^{T} \mathbf{Q}_{D} \mathbf{M}=\left[\begin{array}{lll}
0 & 0 & 0 \\
0 & 2 & 0 \\
0 & 0 & 2
\end{array}\right]
$$


Conversely, any real solution $\mathbf{M}$ to (3.5) with a nonzero first column is the curvaturecenter coordinate matrix $\mathbf{M}_{\mathcal{D}}$ of a unique ordered, oriented Descartes configuration $\mathcal{D}$.

Remark. The hypothesis of a nonzero first column is necessary in Theorem 3.1. Given the matrix $\mathbf{M}$ of a Descartes configuration, the matrix $\tilde{\mathbf{M}}$ obtained by zeroing out its first column will continue to satisfy (3.5), and will not come from a Descartes configuration.

Proof. An $n$-dimensional version of this theorem was stated as Theorem 3.2 in [28], and the "if" direction of the result was proved there. (The converse part of the theorem was not proved there.) More precisely, in [28] it was proved that the curvature-center coordinate matrix $\mathbf{M}$ of any ordered, oriented Descartes configuration satisfies (3.5). The first column of the matrix $\mathbf{M}$ of a Descartes configuration cannot be identically zero because at least two circles in any Descartes configuration have nonzero curvature.

To establish the converse part of the theorem, we need Theorem 3.2 below, which was independently proved in [28]. We postpone the proof to the end of Section 3.1.

The curvature-center coordinates $\mathbf{m}(C)$ uniquely determine a circle in general position, but they fail to be unique for the degenerate case of a line, where the information $(0, \mathbf{h})$ determines only a direction normal to the line and not its position.

In [28] three of the authors defined augmented curvature-center coordinates $\mathbf{w}(C)$ of an oriented circle $C$ with signed curvature $b$ and center $(x, y)$, which resolve this ambiguity. These represent $C$ by a row vector

$$
\mathbf{w}(C):=\left(\bar{b}, b, b x_{1}, b x_{2}\right)
$$

in which $\bar{b}$ is the signed curvature of the oriented circle obtained by inversion in the unit circle. The operation of inversion in the unit circle acts on $\mathbb{R}^{2}$ by

$$
(x, y) \mapsto\left(x^{\prime}, y^{\prime}\right)=\left(\frac{x}{x^{2}+y^{2}}, \frac{y}{x^{2}+y^{2}}\right) .
$$

It maps a circle $C$ of finite (oriented) radius $r$ to the circle $\bar{C}$ with center $\overline{\mathbf{x}}=\mathbf{x} /\left(|\mathbf{x}|^{2}-r^{2}\right)$ and oriented radius $\bar{r}=r /\left(|\mathbf{x}|^{2}-r^{2}\right)$, having oriented curvature $\bar{b}=\left(|\mathbf{x}|^{2}-r^{2}\right) / r$. If $C$ is a straight line with specified normal direction, we determine $\bar{C}$ as the image of this line, with orientation coming from the specified normal. In the degenerate case that $\bar{C}$ is a straight line we define $\bar{b}=0$. In all cases,

$$
(b x, b y)=\frac{\mathbf{x}}{r}=\frac{\overline{\mathbf{x}}}{\bar{r}}=\left(\bar{b} x^{\prime}, \bar{b} y^{\prime}\right),
$$

so that $C$ and $\bar{C}$ have the same curvature $\times$ center data.

Augmented curvature-center coordinates provide a global coordinate system: no two distinct oriented circles have the same coordinates. The only case to resolve is when $C$ is a straight line, i.e. $b=0$. The relation (3.7) shows that $(\bar{b}, b x, b y)$ are the curvaturecenter coordinates of $\bar{C}$, and if $\bar{b} \neq 0$, this uniquely determines $\bar{C}$; inversion in the unit circle then determines $C$. In fact, $\bar{b}$ in this case is twice the distance of $C$ from the origin. In the remaining case, $b=\bar{b}=0$ and $C=\bar{C}$ is the unique line passing through the origin whose unit normal is given by the remaining coordinates. 
Given a collection $\left(C_{1}, C_{2}, C_{3}, C_{4}\right)$ of four oriented circles (possibly lines) in $\mathbb{R}^{2}$, the augmented matrix $\mathbf{W}$ associated with it is the $4 \times 4$ matrix whose $j$ th row has entries given by the augmented curvature-center coordinates $\mathbf{w}\left(C_{j}\right)$ of the $j$ th circle. The following result characterizes oriented Descartes configurations.

Theorem 3.2 (Augmented Euclidean Descartes Theorem). Given an ordered configuration $\mathcal{D}$ offour oriented circles (or lines) $\left\{C_{i}: 1 \leq i \leq 4\right\}$ with curvatures $\left(b_{1}, b_{2}, b_{3}, b_{4}\right)$ and centers $\left\{\left(x_{i}, y_{i}\right): 1 \leq i \leq 4\right\}$, let $\mathbf{W}_{\mathcal{D}}$ be the $4 \times 4$ matrix

$$
\mathbf{W}_{\mathcal{D}}:=\left[\begin{array}{llll}
\bar{b}_{1} & b_{1} & b_{1} x_{1} & b_{1} y_{1} \\
\bar{b}_{2} & b_{2} & b_{2} x_{2} & b_{2} y_{2} \\
\bar{b}_{3} & b_{3} & b_{3} x_{3} & b_{3} y_{3} \\
\bar{b}_{4} & b_{4} & b_{4} x_{4} & b_{4} y_{4}
\end{array}\right] .
$$

If $\mathcal{D}$ is an (ordered, oriented) Descartes configuration then $\mathbf{W}_{\mathcal{D}}$ satisfies

$$
\mathbf{W}^{T} \mathbf{Q}_{D} \mathbf{W}=\left[\begin{array}{rrrr}
0 & -4 & 0 & 0 \\
-4 & 0 & 0 & 0 \\
0 & 0 & 2 & 0 \\
0 & 0 & 0 & 2
\end{array}\right]
$$

Conversely, any real solution $\mathbf{W}$ to (3.9) is the augmented matrix $\mathbf{W}_{\mathcal{D}}$ of a unique ordered, oriented Descartes configuration $\mathcal{D}$.

Proof. This is proved as the two-dimensional case of Theorem 3.3 in [28].

We call the quadratic form $Q_{W}$ defined by the matrix

$$
\mathbf{Q}_{W}=\left[\begin{array}{rrrr}
0 & -4 & 0 & 0 \\
-4 & 0 & 0 & 0 \\
0 & 0 & 2 & 0 \\
0 & 0 & 0 & 2
\end{array}\right]
$$

the Wilker quadratic form. We name this quadratic form after J. B. Wilker [42], who introduced in spherical geometry a coordinate system analogous to augmented curvaturecenter coordinates, see Section 2, pp. 388-390, and Section 9 in [42]. However, Wilker did not explicitly formulate any result exhibiting the quadratic form $Q_{W}$, see p. 349 in [28].

Theorem 3.2 identifies the set of all ordered, oriented Descartes configurations $\mathbb{D}$ with the set $\mathcal{M}_{\mathbb{D}}$ of real solutions $\mathbf{W}=\mathbf{W}_{\mathcal{D}}$ to the matrix equation (3.9). This equation states that the augmented matrix coordinates of an oriented Descartes configuration give an intertwining map between the Descartes form and the Wilker form. The set $\mathcal{M}_{\mathbb{D}}$ has the structure of a six-dimensional affine real-algebraic variety.

It is possible to refine the parameter space to a moduli space $\tilde{\mathcal{M}}_{\mathbb{D}}$ of (unordered, unoriented) Descartes configurations as an orbifold $\mathcal{M}_{\mathbb{D}} / \sim$ obtained by quotienting by a finite group of order 48 (acting on the left). This group is generated by the $4 \times 4$ permutation matrices (permuting rows) and $-\mathbf{I}$, which reverses total orientation. This 
orbifold has singular points at those ordered, oriented Descartes configurations which remain invariant under a nontrivial permutation matrix. For our purposes it is more convenient to use the parameter space $\mathcal{M}_{\mathbb{D}}$ which is a smooth manifold.

Both the Descartes quadratic form and the Wilker quadratic form are equivalent over the real numbers to the Lorentz quadratic form $Q_{\mathcal{L}}(x):=-x_{0}^{2}+x_{1}^{2}+x_{2}^{3}+x_{3}^{2}$, with associated matrix

$$
\mathbf{Q}_{\mathcal{L}}=\left[\begin{array}{rrrr}
-1 & 0 & 0 & 0 \\
0 & 1 & 0 & 0 \\
0 & 0 & 1 & 0 \\
0 & 0 & 0 & 1
\end{array}\right]
$$

For any quadratic form $Q$, let $\operatorname{Aut}(Q)$ be the group of automorphisms under the "congruence action," defined by

$$
\operatorname{Aut}(Q)=\left\{\mathbf{U} \in G L(4, \mathbb{R}): \mathbf{U}^{T} \mathbf{Q} \mathbf{U}=\mathbf{Q}\right\},
$$

where $\mathbf{Q}$ is the symmetric matrix that represents $Q$. The Lorentz quadratic form has a large group of automorphisms $\operatorname{Aut}\left(Q_{\mathcal{L}}\right)$, which is exactly the real Lorentz group $O(3,1)$.

The Descartes form and Wilker form are not only equivalent to the Lorentz form over the real numbers, but also over the rational numbers. For the Descartes form one has

$$
\mathbf{Q}_{D}=\mathbf{J}_{0}^{T} \mathbf{Q}_{\mathcal{L}} \mathbf{J}_{0}
$$

in which

$$
\mathbf{J}_{0}=\frac{1}{2}\left[\begin{array}{rrrr}
1 & 1 & 1 & 1 \\
1 & 1 & -1 & -1 \\
1 & -1 & 1 & -1 \\
1 & -1 & -1 & 1
\end{array}\right]
$$

and $\mathbf{J}_{0}=\mathbf{J}_{0}^{T}=\mathbf{J}_{0}^{-1}$. It follows that

$$
\operatorname{Aut}\left(Q_{D}\right)=\mathbf{J}_{0}^{-1} O(3,1) \mathbf{J}_{0}
$$

The rational equivalence of the Wilker quadratic form $Q_{W}$ to the Lorentz form $Q_{\mathcal{L}}$ follows from Theorem 3.2, as soon as we exhibit a Descartes configuration $\mathcal{D}$ whose augmented curvature-center coordinates $\mathbf{W}_{\mathcal{D}}$ are a rational matrix. One is given by

$$
\mathbf{W}_{0}=\mathbf{W}_{\mathcal{D}}=\left[\begin{array}{rrrr}
2 & 0 & 0 & 1 \\
2 & 0 & 0 & -1 \\
0 & 1 & 1 & 0 \\
0 & 1 & -1 & 0
\end{array}\right]
$$

It corresponds to the positively oriented Descartes configuration pictured in Fig. 3, in which the dashed line is the $x$-axis and the two circles touch at the origin.

We then have

$$
\mathbf{Q}_{W}=\mathbf{A}^{T} \mathbf{Q}_{\mathcal{L}} \mathbf{A}
$$




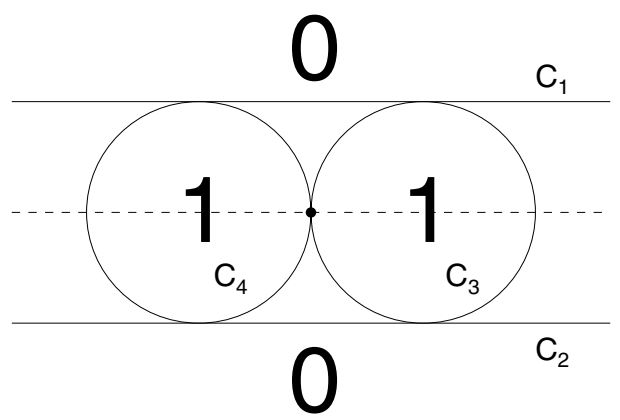

Fig. 3. A strongly integral Descartes configuration.

with

$$
\mathbf{A}=\mathbf{J}_{0} \mathbf{W}_{0}=\left[\begin{array}{rrrr}
2 & 1 & 0 & 0 \\
2 & -1 & 0 & 0 \\
0 & 0 & 1 & 1 \\
0 & 0 & -1 & 1
\end{array}\right]
$$

It follows that

$$
\operatorname{Aut}\left(Q_{W}\right)=\mathbf{A}^{-1} O(3,1) \mathbf{A} .
$$

Finally we note that the determinants of these quadratic forms are given by

$$
\operatorname{det}\left(\mathbf{Q}_{D}\right)=\operatorname{det}\left(\mathbf{Q}_{\mathcal{L}}\right)=-1 \text { and } \operatorname{det}\left(\mathbf{Q}_{W}\right)=-64 .
$$

Taking determinants in (3.9) yields that augmented curvature-center coordinate matrices $\mathbf{W}_{\mathcal{D}}$ have

$$
\operatorname{det}\left(\mathbf{W}_{\mathcal{D}}\right)= \pm 8
$$

Examples show that both values \pm 8 occur.

Proof of the "only if" part of Theorem 3.1. We note that if $\mathbf{M}$ is the curvature-center coordinate matrix of a Descartes configuration, this configuration is unique. Indeed, at least two circles in the configuration have nonzero curvature, so are determined by their curvature-center coordinates. This gives enough information to find the location of any lines in the configuration, and their signed normal vectors are determined uniquely by their curvature-center coordinates. Thus it suffices to show that there exists a $4 \times 4$ matrix W whose last three columns agree with $\mathbf{M}$, such that

$$
\mathbf{W}^{T} \mathbf{Q}_{D} \mathbf{W}=\mathbf{Q}_{W}=\left[\begin{array}{rrrr}
0 & -4 & 0 & 0 \\
-4 & 0 & 0 & 0 \\
0 & 0 & 2 & 0 \\
0 & 0 & 0 & 2
\end{array}\right]
$$

If so, then Theorem 3.2 implies that $\mathbf{W}$ is the augmented curvature-center coordinate matrix of a unique ordered, oriented Descartes configuration $\mathcal{D}$. It follows that $\mathbf{M}$ is the curvature-center coordinate matrix of $\mathcal{D}$. 
This discussion implies that the extension $\mathbf{W}$ is unique, and we proceed to find it by transforming the problem from the Descartes form $\mathbf{Q}_{D}$ to the Lorentz form $\mathbf{Q}_{\mathcal{L}}=$ $\mathbf{J}_{0}^{T} \mathbf{Q}_{D} \mathbf{J}_{0}$. This proof seems "pulled out of thin air" in that it guesses to do the "translation" by the matrix $\mathbf{T}$ below, in terms of which the extended matrix $\tilde{\mathbf{W}}$ has a simple form. We set $\tilde{\mathbf{M}}=\mathbf{J}_{0} \mathbf{M}$ with $\mathbf{J}_{0}$ given by (3.13), so that $\mathbf{J}_{0}=\mathbf{J}_{0}^{T}=\mathbf{J}_{0}^{-1}$. Then

$$
\tilde{\mathbf{M}}^{T} \mathbf{Q}_{\mathcal{L}} \tilde{\mathbf{M}}=\mathbf{M}^{T} \mathbf{Q}_{D} \mathbf{M}=\mathbf{Q}_{0}:=\left[\begin{array}{lll}
0 & 0 & 0 \\
0 & 2 & 0 \\
0 & 0 & 2
\end{array}\right] .
$$

We now note that

$$
\tilde{\mathbf{M}}_{11}=\frac{1}{2}\left(m_{11}+m_{12}+m_{13}+m_{14}\right) \neq 0 .
$$

To verify this, note that the top left entry of (3.5) gives

$Q_{D}\left(m_{11}, m_{12}, m_{13}, m_{14}\right)=\left(m_{11}^{2}+m_{12}^{2}+m_{13}^{2}+m_{14}^{2}\right)-\frac{1}{2}\left(m_{11}+m_{12}+m_{13}+m_{14}\right)^{2}=0$, and if $m_{11}+m_{12}+m_{13}+m_{14}=0$ then we would obtain $m_{11}^{2}+m_{12}^{2}+m_{13}^{2}+m_{14}^{2}=0$, which forces all $m_{1 j}=0$, contradicting the hypothesis. Now there exists a unique matrix

$$
\mathbf{T}=\left[\begin{array}{lll}
a & b & c \\
0 & 1 & 0 \\
0 & 0 & 1
\end{array}\right]
$$

such that the first row of $\tilde{\mathbf{M T}}$ is [1, 0, 0], which takes $a=\left(\tilde{\mathbf{M}}_{11}\right)^{-1}$, so that $\mathbf{T}$ is invertible. We write

$$
\tilde{\mathbf{M}} \mathbf{T}=\left[\begin{array}{ccc}
1 & 0 & 0 \\
v_{21} & v_{22} \sqrt{2} & v_{23} \sqrt{2} \\
v_{31} & v_{32} \sqrt{2} & v_{33} \sqrt{2} \\
v_{41} & v_{42} \sqrt{2} & v_{43} \sqrt{2}
\end{array}\right]
$$

and then have

$$
\mathbf{T}^{T} \tilde{\mathbf{M}}^{T} \mathbf{Q}_{\mathcal{L}} \tilde{\mathbf{M}} \mathbf{T}=\mathbf{T}^{T} \mathbf{Q}_{0} \mathbf{T}=\mathbf{Q}_{0}=\left[\begin{array}{lll}
0 & 0 & 0 \\
0 & 2 & 0 \\
0 & 0 & 2
\end{array}\right] .
$$

This matrix equation is equivalent to the assertion that

$$
\mathbf{V}:=\left[\begin{array}{lll}
v_{21} & v_{22} & v_{23} \\
v_{31} & v_{32} & v_{33} \\
v_{41} & v_{42} & v_{43}
\end{array}\right]
$$

is an orthogonal matrix, i.e. $\mathbf{V}^{T} \mathbf{V}=\mathbf{V} \mathbf{V}^{T}=I$. We now define

$$
\tilde{\mathbf{W}}:=\left[\begin{array}{cccc}
2 & 1 & 0 & 0 \\
-2 v_{21} & v_{21} & v_{22} \sqrt{2} & v_{23} \sqrt{2} \\
-2 v_{31} & v_{31} & v_{32} \sqrt{2} & v_{33} \sqrt{2} \\
-2 v_{41} & v_{41} & v_{42} \sqrt{2} & v_{43} \sqrt{2}
\end{array}\right],
$$


whose last three columns match $\tilde{\mathbf{M T}}$, and verify by direct calculation using (3.23) that

$$
\tilde{\mathbf{W}}^{T} \mathbf{Q}_{\mathcal{L}} \tilde{\mathbf{W}}=\mathbf{Q}_{W} .
$$

We define $\mathbf{W}:=\mathbf{J}_{0}^{-1} \tilde{\mathbf{W}}=\mathbf{J}_{0} \tilde{\mathbf{W}}$, and find that

$$
\mathbf{W}^{T} \mathbf{Q}_{D} \mathbf{W}=\tilde{\mathbf{W}}^{T} \mathbf{J}_{0} \mathbf{Q}_{\mathcal{L}} \mathbf{J}_{0} \tilde{\mathbf{W}}=\mathbf{Q}_{W} .
$$

This is the desired lift, since the last three columns of $\mathbf{W}$ are exactly $\mathbf{M}$.

\subsection{Möbius and Lorentz Group Actions}

The augmented Euclidean Descartes theorem immediately yields two group actions on the space $\mathcal{M}_{\mathbb{D}}$ of ordered, oriented Descartes configurations. The group $\operatorname{Aut}\left(Q_{D}\right)$ acts on the left and the group $\operatorname{Aut}\left(Q_{W}\right)$ acts on the right, as

$$
\mathbf{W}_{\mathcal{D}} \mapsto \mathbf{U W}_{\mathcal{D}} \mathbf{V}^{-1}, \quad \text { with } \quad \mathbf{U} \in \operatorname{Aut}\left(Q_{D}\right), \quad \mathbf{V} \in \operatorname{Aut}\left(Q_{W}\right)
$$

The two group actions clearly commute with each other. Both groups are conjugate to the real Lorentz group $O(3,1)$, and therefore they each have four connected components. These components are specified for any $\mathbf{Y} \in O(3,1)$ by the sign of $\operatorname{det}(\mathbf{Y})$, and by the sign of "total orientation," which is the sign of $\mathbf{Y}_{11}=\mathbf{e}_{1}^{T} \mathbf{Y e}_{1}$, in which $\mathbf{e}_{1}^{T}=(1,0,0,0)$. For $\mathbf{U} \in \operatorname{Aut}\left(Q_{D}\right)$, it is the sign of $\mathbf{1}^{T} \mathbf{U} \mathbf{1}$, in which $\mathbf{1}^{T}=(1,1,1,1)$; and for $\mathbf{V} \in \operatorname{Aut}\left(Q_{W}\right)$, it is the sign of $\mathbf{e}_{1}^{T} \mathbf{A V A}^{-1} \mathbf{e}_{1}$ in which $\mathbf{A}$ is any matrix satisfying $O(3,1)=\mathbf{A} \operatorname{Aut}\left(Q_{W}\right) \mathbf{A}^{-1}$, such as (3.17).

The parameter space $\mathcal{M}_{\mathbb{D}}$ also has four connected components, specified by similar invariants, which are the sign of $\operatorname{det}(\mathbf{W})$ and the total orientation, which is the sign of the $\operatorname{sum}^{3}$ of the (signed) curvatures of the four circles in the Descartes configuration. We let

$$
\mathcal{M}_{\mathbb{D}}=\mathcal{M}_{+}^{\uparrow} \cup \mathcal{M}_{-}^{\uparrow} \cup \mathcal{M}_{+}^{\downarrow} \cup \mathcal{M}_{-}^{\downarrow},
$$

in which the subscript describes the sign of the determinant and the superscript the orientation, with $\uparrow$ being positive orientation. We also let

$$
\mathcal{M}_{\mathbb{D}}^{\uparrow}:=\mathcal{M}_{+}^{\uparrow} \cup \mathcal{M}_{-}^{\uparrow}
$$

denote the set of positively oriented Descartes configurations. The relevance of this decomposition is that the Apollonian group defined in Section 4 leaves the set of positively oriented Descartes configurations $\mathcal{M}_{\mathbb{D}}^{\uparrow}$ invariant.

The action on the right by the elements of $\operatorname{Aut}\left(Q_{W}\right)$ maps circles to circles, since the circles in a Descartes configuration correspond to the rows in the matrix $\mathbf{W}_{\mathcal{D}}$ of an ordered, oriented Descartes configuration. This right action can essentially be identified with the general Möbius group Möb(2) generated by the linear fractional transformations acting on the one-point compactification $\hat{\mathbb{R}}^{2}$ of $\mathbb{R}^{2}$, which is $\operatorname{PSL}(2, \mathbb{C})=S L(2, \mathbb{C}) /\{ \pm \mathbf{I}\}$, together with complex conjugation $\mathbf{z} \mapsto \overline{\mathbf{z}}$. and has two connected components. More

\footnotetext{
${ }^{3}$ The sum of the signed curvatures of the circles of an oriented Descartes configuration cannot be zero.
} 
precisely, $\operatorname{Aut}\left(Q_{W}\right)$ is isomorphic to the direct sum of the Möbius group with a group of order two, which has four connected components, as explained in Appendix A.

The action on the left, by $\operatorname{Aut}\left(Q_{D}\right)$, produces a new oriented Descartes configuration whose coordinates mix together the coordinates of the different circles in the original Descartes configuration. This group action does not make sense as an action on individual circles in the configuration. This group action is intrinsically associated to the sixdimensional space of oriented Descartes configurations.

\section{Theorem 3.3.}

(1) The groups Aut $\left(Q_{D}\right)$ and $\operatorname{Aut}\left(Q_{W}\right)$ are conjugate to $\operatorname{Aut}\left(Q_{\mathcal{L}}\right) \equiv O(3,1)$.

(2) The group $A u t\left(Q_{D}\right)$ acts transitively on the left on the space $\mathcal{M}_{\mathbb{D}}$ of all ordered, oriented Descartes configurations. Given two such Descartes configurations $\mathcal{D}$ and $\mathcal{D}^{\prime}$ there exists a unique $\mathbf{U} \in \operatorname{Aut}\left(Q_{D}\right)$ such that $\mathbf{U} \mathbf{W}_{\mathcal{D}}=\mathbf{W}_{\mathcal{D}^{\prime}}$.

(3) The group $\operatorname{Aut}\left(Q_{W}\right)$ acts transitively on the right on the space of all ordered, oriented Descartes configurations $\mathcal{M}_{\mathbb{D}}$. Given two such Descartes configurations $\mathcal{D}$ and $\mathcal{D}^{\prime}$ there exists a unique $\mathbf{V} \in \operatorname{Aut}\left(Q_{W}\right)$ such that $\mathbf{W}_{\mathcal{D}} \mathbf{V}^{-1}=\mathbf{W}_{\mathcal{D}^{\prime}}$.

(4) The action of $\operatorname{Aut}\left(Q_{D}\right)$ on the space $\mathcal{M}_{\mathbb{D}}$ commutes with the action of $\operatorname{Aut}\left(Q_{W}\right)$.

Remark. The left action by $\operatorname{Aut}\left(Q_{D}\right)$ and the right action by $\operatorname{Aut}\left(Q_{W}\right)$ on $\mathcal{M}_{\mathbb{D}}$ given by Theorem 3.3 can be identified with a left and right action of the Lorentz group $O(3,1)=\operatorname{Aut}\left(Q_{\mathcal{L}}\right)$ on $\mathcal{M}_{\mathbb{D}}$, using (3.14) and (3.18), respectively. Theorem 3.3 shows that both these actions are transitive, and that the stabilizer of a point is the identity element. This is equivalent to saying that the space $\mathcal{M}_{\mathbb{D}}$ is a principal homogeneous space (or torsor) for $O(3,1)$ for either action.

Proof. Part (1) follows from the conjugacy between the Descartes and Wilker forms and the Lorentz form $Q_{\mathcal{L}}$ given in (3.12) and (3.16), respectively. These give

$$
\operatorname{Aut}\left(Q_{D}\right)=\mathbf{J}_{0}^{-1} \operatorname{Aut}\left(Q_{\mathcal{L}}\right) \mathbf{J}_{0} \equiv \mathbf{J}_{0}^{-1} O(3,1) \mathbf{J}_{0}
$$

and

$$
\operatorname{Aut}\left(Q_{W}\right)=\mathbf{A}^{-1} \operatorname{Aut}\left(Q_{\mathcal{L}}\right) \mathbf{A} \equiv \mathbf{A}^{-1} O(3,1) \mathbf{A} .
$$

Parts (2) and (3) follow immediately from (1). Given a fixed $\mathbf{W}=\mathbf{W}_{\mathcal{D}} \in \mathcal{M}_{\mathbb{D}}$, we assert that

$$
\mathcal{M}_{\mathbb{D}}=\operatorname{Aut}\left(Q_{D}\right) \mathbf{W}
$$

Taking $\mathbf{W}_{0}$ in (3.15) we have

$$
\left(\mathbf{W} \mathbf{W}_{0}^{-1}\right)^{T} \mathbf{Q}_{D}\left(\mathbf{W} \mathbf{W}_{0}^{-1}\right)=\left(\mathbf{W A}^{-1} \mathbf{J}_{0}\right)^{T} \mathbf{Q}_{D}\left(\mathbf{W} \mathbf{A}^{-1} \mathbf{J}_{0}\right)=\mathbf{Q}_{D},
$$

so that $\mathbf{W W}_{0}^{-1} \in \operatorname{Aut}\left(Q_{D}\right)$ and $\operatorname{Aut}\left(Q_{D}\right) \mathbf{W} \mathbf{W}_{0}^{-1}$ forms a single orbit of $\operatorname{Aut}\left(Q_{D}\right)$. The map $\mathbf{W}_{\mathcal{D}} \mapsto \mathbf{W}_{\mathcal{D}} \mathbf{W}_{0}^{-1}$ from $\mathcal{M}_{\mathbb{D}}$ into $\operatorname{Aut}\left(Q_{D}\right)$ is one-to-one since $\mathbf{W}_{0}$ is invertible, and it is onto since the domain includes $\operatorname{Aut}\left(Q_{D}\right) \mathbf{W}$. Thus (3.24) follows, and this gives (2). We similarly obtain

$$
\mathcal{M}_{\mathbb{D}}=\mathbf{W} \operatorname{Aut}\left(Q_{W}\right)^{-1}=\mathbf{W} \operatorname{Aut}\left(Q_{W}\right),
$$

which gives (3). 
Finally, part (4) follows directly from parts (2) and (3), since in general a left and a right action of two matrix groups on a space of matrices commute.

In this paper we mainly study structures associated to the left action of $\operatorname{Aut}\left(Q_{D}\right)$ on $\mathcal{M}_{\mathbb{D}}$. We term the left action the Lorentz action, although this is a misnomer, since the right action can also be identified with a Lorentz group action. However, we wish to assign different names for the two actions, and the right action is conveniently called the Möbius action, because of its relation to a Möbius group action on circles on the Riemann sphere, detailed in Appendix A.

\subsection{Distinguished Elements of Aut $\left(Q_{D}\right)$}

We now describe some specific elements of $\operatorname{Aut}\left(Q_{D}\right)$ that have a nice geometrically interpretable action on every Descartes configuration, visualizable in terms of inversions, and whose associated matrices have integer (or half-integer) entries.

The first set of four operations correspond to inversion in the circle determined by the three intersection points of circles in a Descartes configuration $\mathcal{D}$ that avoid one particular circle. There are four possibilities for these. This inversion fixes the three circles involved in the intersections and moves the fourth circle, to the unique other circle that is tangent to the first three circles. For this reason we call it the reflection operator. Let $\mathfrak{s}_{1}=\mathfrak{s}_{1}[\mathcal{D}]$ denote the Möbius transformation of this kind that moves the circle $C_{1}$. In particular, $\mathfrak{s}_{1}$ maps the Descartes configuration $\mathcal{D}=\left(C_{1}, C_{2}, C_{3}, C_{4}\right)$ to $\mathfrak{s}_{1}(\mathcal{D})=\left(C_{1}^{\prime}, C_{2}, C_{3}, C_{4}\right)$. This reflection operator depends on the specific Descartes configuration. However, for all Descartes configurations $\mathcal{D}$ there holds

$$
\mathbf{W}_{\mathfrak{s}_{1}(\mathcal{D})}=\mathbf{S}_{1} \mathbf{W}_{\mathcal{D}}
$$

where $\mathbf{S}_{1} \in \operatorname{Aut}\left(Q_{D}\right)$ is given by

$$
\mathbf{S}_{1}=\left[\begin{array}{rrrr}
-1 & 2 & 2 & 2 \\
0 & 1 & 0 & 0 \\
0 & 0 & 1 & 0 \\
0 & 0 & 0 & 1
\end{array}\right]
$$

belongs to $\operatorname{Aut}\left(Q_{D}\right)$ and is independent of the location of $\mathcal{D}$. The geometric action of $\mathfrak{s}_{1}$ is pictured in Fig. 4. The other three operations $\mathfrak{s}_{2}, \mathfrak{s}_{3}, \mathfrak{s}_{4}$ give similar matrices $\mathbf{S}_{2}, \mathbf{S}_{3}, \mathbf{S}_{4}$, obtained by permuting the first and $j$ th rows and columns of $\mathbf{S}_{1}$ for $2 \leq j \leq 4$.

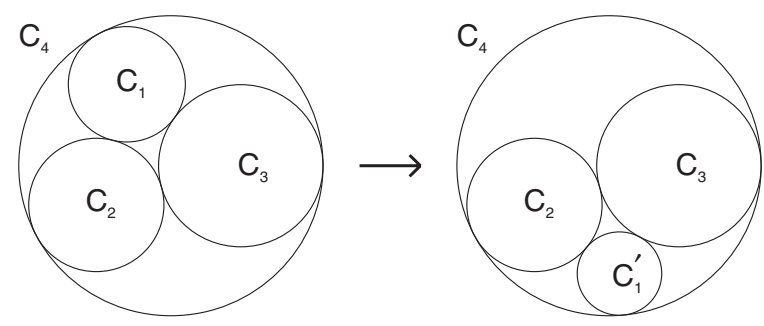

Fig. 4. The reflection operator $\mathfrak{s}_{1}$. 
To prove (3.25) we check that $\mathbf{S}_{1} \in \operatorname{Aut}\left(Q_{D}\right)$. Then for any Descartes configuration $\mathcal{D}$ Theorem 3.2 gives that $\mathbf{S}_{1} \mathbf{W}_{\mathcal{D}}=\mathbf{W}_{\mathcal{D}^{\prime}}$ for some Descartes configuration $\mathcal{D}^{\prime}$. The configuration $\mathcal{D}^{\prime}$ necessarily has three oriented circles fixed, with the fourth moved, so the fourth one must be the unique other circle tangent to the given three, and its orientation is uniquely determined by the other three orientations. Now $\mathbf{W}_{\mathfrak{S}_{1}(\mathcal{D})}$ corresponds to that Descartes configuration consisting of the same four ordered circles, which has the same (positive or negative) orientation as $\mathcal{D}^{\prime}$.

A second set of four operations corresponds to inversion in one of the four circles in a Descartes configuration. Let that circle be $C_{1}$. Now $C_{1}$ remains fixed, while the other three circles change. Denoting this inversion by $\mathfrak{s}_{1}^{\perp}$, it is easy to show that

$$
\mathbf{W}_{\mathfrak{s}_{1}^{\perp}(\mathcal{D})}=\mathbf{S}_{1}^{\perp} \mathbf{W}_{\mathcal{D}},
$$

where $\mathbf{S}_{1}^{\perp} \in \operatorname{Aut}\left(Q_{D}\right)$ is given by

$$
\mathbf{S}_{1}^{\perp}=\left[\begin{array}{rrrr}
-1 & 0 & 0 & 0 \\
2 & 1 & 0 & 0 \\
2 & 0 & 1 & 0 \\
2 & 0 & 0 & 1
\end{array}\right] .
$$

The corresponding matrices $\mathbf{S}_{2}^{\perp}, \mathbf{S}_{3}^{\perp}, \mathbf{S}_{4}^{\perp}$ are obtained by permuting the first and $j$ th rows and columns of $\mathbf{S}_{1}^{\perp}$ for $2 \leq j \leq 4$. The operation $\mathfrak{s}_{1}^{\perp}$ is pictured in Fig. 5 .

Finally we describe an operator, which we call the duality operator $\mathbf{D}$, and which is an involution, as follows. Given a Descartes configuration $\mathcal{D}$, there exists a Descartes configuration $\mathcal{D}^{\prime}$ obtained from it which consists of the four circles each of which passes through the three intersection points avoiding one circle. The resulting configuration has the same six points of tangency as the original configuration, and the circles in the configuration $\mathcal{D}^{\prime}$ are perpendicular to the circles of $\mathcal{D}$ at these tangency points. This is pictured in Fig. 6.

Straightforward computation shows that

$$
\mathbf{W}_{\mathcal{D}^{\prime}}=\mathbf{D W}_{\mathcal{D}},
$$
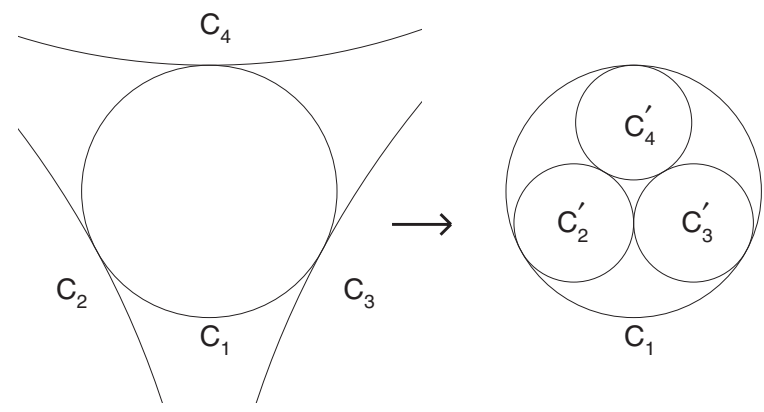

Fig. 5. The inversion operation $\mathfrak{s}_{1}^{\perp}$. 


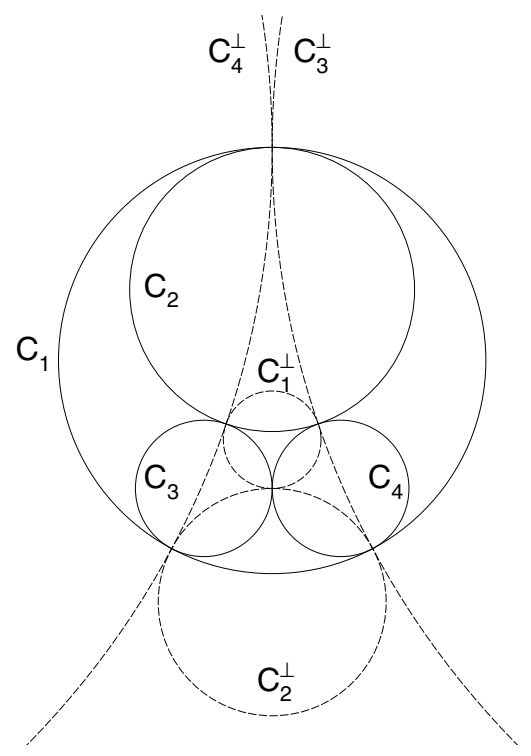

Fig. 6. The dual operation.

with

$$
\mathbf{D}=\frac{1}{2}\left[\begin{array}{rrrr}
-1 & 1 & 1 & 1 \\
1 & -1 & 1 & 1 \\
1 & 1 & -1 & 1 \\
1 & 1 & 1 & -1
\end{array}\right] .
$$

Note that $\mathbf{D}=-\mathbf{Q}_{D} \in \operatorname{Aut}\left(Q_{D}\right)$. There is a Möbius transformation $\mathfrak{d}=\mathfrak{d}_{\mathcal{D}}$ which sends $\mathcal{D} \mapsto \mathfrak{d}(\mathcal{D})=\mathcal{D}^{\prime}$, and it depends on $\mathcal{D}$.

\section{Apollonian Packings and the Apollonian Group}

Apollonian circle packings are infinite packings of circles recursively constructed from a given positively oriented Descartes configuration $\mathcal{D}$. For simplicity consider a positively oriented Descartes configuration $\mathcal{D}=\left\{C_{1}, C_{2}, C_{3}, C_{4}\right\}$ in which circle $C_{4}$ encloses the other three, so that the interior of $C_{4}$ includes the point $\mathbf{z}_{\infty}$ at infinity. The uncovered area consists of four lunes, indicated by the shading in Fig. 7.

The zeroth stage packing $\mathcal{P}_{\mathcal{D}}^{(0)}=\mathcal{D}$. At the first stage we inscribe a circle in each lune, to obtain a circle packing $\mathcal{P}_{\mathcal{D}}^{(1)}$ containing eight circles. Each of these circles lies in a unique (unordered) Descartes configuration in the first stage packing, consisting of it and the three circles it touches. The uncovered area that remains consists of 12 lunes. See Fig. 8.

At the second stage we inscribe a circle in each of these lunes, which produces the second stage packing $\mathcal{P}_{\mathcal{D}}^{(2)}$. Continuing in this way, at the $n$th stage we add $4 \cdot 3^{n-1}$ circles, and each of these lies in a unique Descartes configuration in the $n$th stage partial 


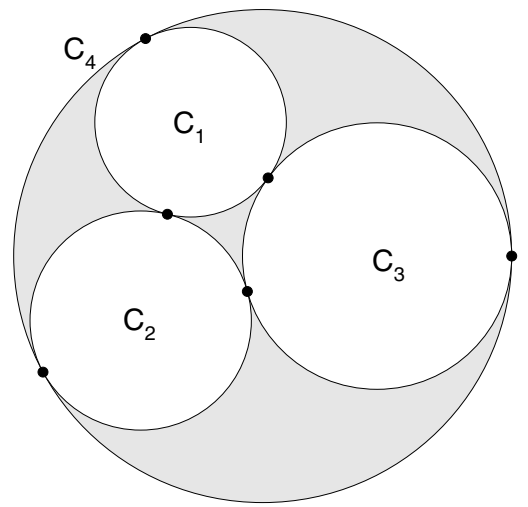

Fig. 7. A zeroth stage packing $\mathcal{P}_{\mathcal{D}}^{(0)}$ and uncovered regions (lunes).

packing. The Apollonian packing $\mathcal{P}_{\mathcal{D}}$ associated to $\mathcal{D}$ is the limit packing

$$
\mathcal{P}_{\mathcal{D}}:=\bigcup_{n=1}^{\infty} \mathcal{P}_{\mathcal{D}}^{(n)}
$$

One can regard an Apollonian packing $\mathcal{P}$ as a geometric object consisting of an infinite collection of circles. These circles are described as the four orbits of a group of Möbius transformations $G_{\mathcal{A}}(\mathcal{D})$ acting on the circles $\left(C_{1}, C_{2}, C_{3}, C_{4}\right)$ in original Descartes configuration $\mathcal{D}$. The group

$$
G_{\mathcal{A}}(\mathcal{D}):=\left\langle\mathfrak{s}_{1}, \mathfrak{s}_{2}, \mathfrak{s}_{3}, \mathfrak{s}_{4}\right\rangle
$$

in which $\mathfrak{s}_{j}$ is the inversion with respect to the circle that passes through the three intersection points of $\mathcal{D}$ that do not include the circle $C_{j}$, as indicated in Section 3.3. One can check that the circles added at the $n$th stage of the construction above correspond to words $\mathfrak{s}_{i_{1}} \mathfrak{s}_{i_{2}} \cdots \mathfrak{s}_{i_{n}}$ of length $n$ in the generators of this group, in which any two adjacent generators are distinct, i.e. $i_{j} \neq i_{j+1}$.

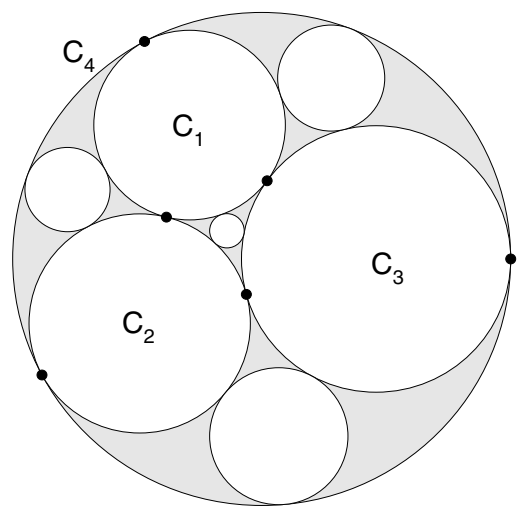

Fig. 8. A first stage packing $\mathcal{P}_{\mathcal{D}}^{(1)}$. 
The key geometric property of Apollonian circle packings is that they can be viewed as packings of disks having the circles as boundary. To each circle in the Riemann sphere correspond two disks having the circle as boundary. Recall that the "interior" of an oriented circle corresponds to making a choice of one of these two disks, indicated by a choice of sign of the curvature, when it is nonzero (and by a choice of normal vector if the curvature is zero). The disk packing property of an Apollonian packing corresponds to treating its Descartes configurations as positively oriented.

Theorem 4.1. For any Apollonian packing generated by a positively oriented Descartes configuration, the interiors of all circles in the packing are disjoint.

Proof. We generate the packing from a single Descartes configuration $\mathcal{D}$ chosen to have positive total orientation, so that the interiors of its four circles defined by the total orientation are disjoint. Positive total orientation is preserved by the action of Möbius transformations; see Theorem A.1 in Appendix A. Let

$$
\mathcal{P}_{\mathcal{D}}[m]:=\bigcup_{n=1}^{m} \mathcal{P}_{\mathcal{D}}^{(n)},
$$

where $\mathcal{D}$ is a positively oriented Descartes configuration. We prove by induction on $m$ the following statement: $\mathcal{P}_{\mathcal{D}}[m]$ consists of $2\left(3^{m}+1\right)$ circles which form the boundary of $2\left(3^{m}+1\right)$ disks and $4 \cdot 3^{m}$ lune areas. The interiors of the disks, as well as the lunes, are mutually disjoint.

The base case $m=0$ is easy. Since any two positively oriented Descartes configurations are equivalent under the Möbius transformations, which map circles to circles and preserve the (total) orientation (see Theorem A.2, Appendix A), we can simply check the Descartes configuration given in Fig. 7, which has four circles that form the boundary of four disks and four lunes with disjoint interiors.

Assume the statement holds for $m \geq 0$. We carry out the inductive step for $m+1$. By the inductive construction above,

$$
\mathcal{P}_{\mathcal{D}}[m+1]=\mathcal{P}_{\mathcal{D}}[m] \cup \mathcal{P}_{\mathcal{D}}^{(m+1)} .
$$

The circles of $\mathcal{P}_{\mathcal{D}}[m]$ remain in $\mathcal{P}_{\mathcal{D}}[m+1]$ with their interiors untouched. In each lune of $\mathcal{P}_{\mathcal{D}}[m]$ a new circle is inscribed, breaking the original lune into four parts—one disk and three smaller lunes, with disjoint interiors. Combining the inductive hypothesis, we have that $\mathcal{P}_{\mathcal{D}}[m+1]$ consists of $2\left(3^{m}+1\right)+4 \cdot 3^{m}=2\left(3^{m+1}+1\right)$ many circles with empty interiors, and the uncovered area are $3 \cdot 4 \cdot 3^{m}=4 \cdot 3^{m+1}$ lunes with disjoint interiors. Each new Descartes configuration shares the interior of three circles with the Descartes configuration generating it; this implies it has the same orientation as the preceding one; hence it has positive total orientation. This proves the statement for $m+1$.

We first consider an Apollonian packing as a geometric object. A geometric Apollonian packing $\mathcal{P}$ is the point set consisting of a countable collection of circles on the Riemann sphere $\hat{\mathbb{C}}=\mathbb{C} \cup\{\infty\}$ obtained by the construction above. It has Hausdorff dimension 1 , since it is a countable union of sets of Hausdorff dimension 1 (circles). Let 
$G(\mathcal{P})$ denote the group of Möbius transformations that leave $\mathcal{P}$ invariant. This group can be proved to be a discrete subgroup of the group of all Möbius transformations, which acts transitively on the circles in the packing. The group $G(\mathcal{P})$ contains $G_{\mathcal{A}}(\mathcal{D})$ above as a subgroup of index 24 , with cosets given by 24 Möbius transformations whose effect is to fix a generating Descartes configuration $\mathcal{D}$ and permute the four circles in it. (These are nontrivial facts, and we do not prove them here.)

The geometric Apollonian packing is not a closed set on the Riemann sphere. We define $\Lambda(\mathcal{P})$ to be its closure on the Riemann sphere, and call it the residual set of the Apollonian packing. These sets are prototypical examples of fractal sets, and have been much studied; in 1967 Hirst [25] showed these sets have Hausdorff dimension strictly between 1 and 2 . For later use, we summarize properties of $\Lambda(\mathcal{P})$ in the following result.

Theorem 4.2. The residual set $\Lambda(\mathcal{P})$ of a geometric Apollonian packing $\mathcal{P}$ has the following properties:

(1) $\Lambda(\mathcal{P})$ is the complement in the Riemann sphere of the interiors of all circles in the packing. Here "interior" is defined by a positive orientation of a Descartes configuration in the packing.

(2) $\Lambda(\mathcal{P})$ has a Hausdorff dimension which is independent of the packing $\mathcal{P}$, that satisfies the bounds

$$
1.300197<\operatorname{dim}_{H}(\Lambda(\mathcal{P}))<1.314534 .
$$

In particular, $\Lambda(\mathcal{P})$ has Lebesgue measure zero.

(3) $\Lambda(\mathcal{P})$ is the closure of the countable set of all tangency points of circles in the packing.

(4) $\Lambda(\mathcal{P})$ is invariant under the action of the discrete group $G(\mathcal{P})$ of Möbius transformations.

Remark. Property (1) is the more usual definition of the residual set of an Apollonian packing.

Proof. Let $I(\mathcal{P})$ be the open set which is the union of the interiors of all circles in the packing. It is clear that

$$
\Lambda(\mathcal{P}) \subset \Lambda^{*}(\mathcal{P}):=\hat{\mathbb{C}} \backslash I(\mathcal{P}) .
$$

The set $\Lambda^{*}(\mathcal{P})$ is the usual definition of the residual set of an Apollonian packing; we will show $\Lambda(\mathcal{P})=\Lambda^{*}(\mathcal{P})$.

The result of Hirst [25] that the Hausdorff dimension of $\Lambda^{*}(\mathcal{P})$ is strictly less than two implies that $\Lambda^{*}(\mathcal{P})$ has Lebesgue measure zero. The sharper bounds on the Hausdorff dimension stated here were obtained in 1973 by Boyd [7]. A later result of Boyd [9] suggests that $\operatorname{dim}_{H}\left(\Lambda^{*}(\mathcal{P})\right)=1.3056 \pm 0.0001$. Thomas and Dhar [40] give a nonrigorous approximation scheme suggesting that this Hausdorff dimension is 1.30568673 with an error of 1 in the last digit. See pp. 125-131 in [18] for proofs of $1<\operatorname{dim}_{H}\left(\Lambda^{*}(\mathcal{P})\right)<1.432$.

Now let $\mathbf{z} \in \Lambda^{*}(\mathcal{P})$. The interiors of the circles in the Apollonian packing cover all but a measure zero area in a neighborhood of $\mathbf{z}$. This requires infinitely many circles, so their radii must go to zero, since there are only finitely many circles of radius larger than 
any positive bound. Since each of these circles has a tangency point on it, the point $\mathbf{z}$ is a limit point of such tangency points. Since the tangency points are contained in $\mathcal{P}$, so we conclude that $\Lambda^{*}(\mathcal{P}) \subset \Lambda(\mathcal{P})$. This gives $\Lambda(\mathcal{P})=\Lambda^{*}(\mathcal{P})$, at which point properties (1)-(3) follow.

Property (4) follows by observing that the group invariance of $\mathcal{P}$ carries over to its closure, by applying it to any Cauchy sequence of points in $\mathcal{P}$.

The main viewpoint of this paper is to treat an Apollonian circle packing $\mathcal{P}$ as described "algebraically" by the set of all Descartes configurations it contains, which we denote $\mathbb{D}(\mathcal{P})$. In the construction process above, except for the four circles in $\mathcal{D}$, each other circle $C$ in the packing corresponds to the unique (unordered) Descartes configuration containing it which occurs at the stage of the construction where $C$ first appears. Each (unordered, unoriented) Descartes configuration appears exactly once in this correspondence, except for the base configuration $\mathcal{D}$ which corresponds to the initial four circles. These Descartes configurations can be described in terms of orbits of a discrete subgroup $\mathcal{A}$ of $\operatorname{Aut}\left(Q_{\mathcal{D}}\right)$, called here the Apollonian group.

The Apollonian group $\mathcal{A}$ is a subgroup of $\operatorname{Aut}\left(Q_{D}\right)$ defined by the action of the reflection operations on Descartes configurations described in Section 3.3. These correspond to inversions in the circles passing through the three tangency points in a Descartes configuration that do not include one fixed circle in the configuration.

Definition 4.1. The Apollonian group $\mathcal{A}$ is the subgroup of $\operatorname{Aut}\left(Q_{D}\right)$ defined by

$$
\mathcal{A}:=\left\langle\mathbf{S}_{1}, \mathbf{S}_{2}, \mathbf{S}_{3}, \mathbf{S}_{4}\right\rangle,
$$

where

$$
\begin{aligned}
\mathbf{S}_{1}=\left[\begin{array}{rrrr}
-1 & 2 & 2 & 2 \\
0 & 1 & 0 & 0 \\
0 & 0 & 1 & 0 \\
0 & 0 & 0 & 1
\end{array}\right], & \mathbf{S}_{2}=\left[\begin{array}{rrrr}
1 & 0 & 0 & 0 \\
2 & -1 & 2 & 2 \\
0 & 0 & 1 & 0 \\
0 & 0 & 0 & 1
\end{array}\right], \\
\mathbf{S}_{3}=\left[\begin{array}{rrrr}
1 & 0 & 0 & 0 \\
0 & 1 & 0 & 0 \\
2 & 2 & -1 & 2 \\
0 & 0 & 0 & 1
\end{array}\right], & \mathbf{S}_{4}=\left[\begin{array}{rrrr}
1 & 0 & 0 & 0 \\
0 & 1 & 0 & 0 \\
0 & 0 & 1 & 0 \\
2 & 2 & 2 & -1
\end{array}\right] .
\end{aligned}
$$

We now characterize the set of ordered, oriented Descartes configurations $\mathbb{D}(\mathcal{P})$ in an Apollonian circle packing $\mathcal{P}$ in terms of this group. We note that a single unordered, unoriented Descartes configuration, corresponds to exactly 48 ordered, oriented Descartes configuration, since there are 24 choices of ordering of the four circles, and two choices of orientation.

Theorem 4.3. The ordered, oriented Descartes configurations in an Apollonian circle packing comprise a union of 48 orbits of the Apollonian group. Each of these orbits contains exactly one ordered, oriented representative of each (unordered, unoriented) Descartes configuration in the packing. 
Proof. Let $\mathcal{D}=\left(C_{1}, C_{2}, C_{3}, C_{4}\right)$ be the zeroth stage packing of $\mathcal{P}$, which is ordered and positively oriented. It is enough to show that $\mathcal{A}(\mathcal{D})$ contains exactly one ordered, positively oriented representative of each unordered, unoriented Descartes configuration in the packing. Note that $\mathbf{S}_{1}^{2}=\mathbf{S}_{2}^{2}=\mathbf{S}_{3}^{2}=\mathbf{S}_{4}^{2}=\mathbf{I}$, and for any word $\mathbf{S}_{i_{1}} \mathbf{S}_{i_{2}} \cdots \mathbf{S}_{i_{n}}\left(i_{j} \neq i_{j+1}\right)$, the Descartes configuration $\mathcal{D}^{\prime}=\left(C_{1}^{\prime}, C_{2}^{\prime}, C_{3}^{\prime}, C_{4}^{\prime}\right)$ with augmented curvature-center coordinates $\mathbf{W}_{\mathcal{D}^{\prime}}=\mathbf{S}_{i_{1}} \mathbf{S}_{i_{2}} \cdots \mathbf{S}_{i_{n}} \mathbf{W}_{\mathcal{D}}$ is a positively oriented Descartes configuration containing a unique circle $C_{i_{n}}^{\prime}$ in the $n$th stage partial packing. Since no two circles at level $n$ touch for $n \geq 1$, by induction it is easy to see that

$$
\mathcal{A}=\left\{\mathbf{S}_{i_{1}} \mathbf{S}_{i_{2}} \cdots \mathbf{S}_{i_{n}} \mid n \geq 0, i_{j} \neq i_{j+1}\right\}
$$

and

$$
\mathcal{A}(\mathcal{D})=\left\{\mathcal{D}^{\prime} \mid \mathbf{W}_{\mathcal{D}^{\prime}}=\mathbf{S}_{i_{1}} \mathbf{S}_{i_{2}} \cdots \mathbf{S}_{i_{n}} \mathbf{W}_{\mathcal{D}}, n \geq 0, i_{j} \neq i_{j+1}\right\} .
$$

Furthermore, for any ordered, positively oriented configuration $\mathcal{D}^{\prime \prime}$ in $\mathcal{P}$, there is a unique permutation $\sigma$ such that $\sigma\left(\mathcal{D}^{\prime \prime}\right)=\mathcal{D}^{\prime} \in \mathcal{A}(\mathcal{D})$, where there is a unique sequence $i_{1} i_{2} \cdots i_{n}, 1 \leq i_{j} \leq 4$ and $i_{j} \neq i_{j+1}$, such that $\mathbf{W}_{\mathcal{D}^{\prime}}=\mathbf{S}_{i_{1}} \mathbf{S}_{i_{2}} \cdots \mathbf{S}_{i_{n}} \mathbf{W}_{\mathcal{D}}$. This proves that all the ordered, positively oriented Descartes configurations in an Apollonian circle packing comprise a union of 24 orbits of the Apollonian group. The theorem follows by counting both the positively and negatively oriented Descartes configurations.

Theorem 4.3 shows that the orbit under the Apollonian group of a single (ordered, oriented) Descartes configuration completely describes an Apollonian packing.

\section{Dual Apollonian Group}

As explained in Section 3, the operation of inversion in the each of the individual circles of a Descartes configuration is described by an integral matrix in $\operatorname{Aut}\left(Q_{D}\right)$. This leads us to the following definition.

Definition 5.1. The dual Apollonian group $\mathcal{A}^{\perp}$ is the subgroup of $\operatorname{Aut}\left(Q_{D}\right)$ generated by the matrices

$$
\begin{array}{rlrl}
\mathbf{S}_{1}^{\perp} & =\left[\begin{array}{rrrr}
-1 & 0 & 0 & 0 \\
2 & 1 & 0 & 0 \\
2 & 0 & 1 & 0 \\
2 & 0 & 0 & 1
\end{array}\right], & \mathbf{S}_{2}^{\perp}= & {\left[\begin{array}{rrrr}
1 & 2 & 0 & 0 \\
0 & -1 & 0 & 0 \\
0 & 2 & 1 & 0 \\
0 & 2 & 0 & 1
\end{array}\right],} \\
\mathbf{S}_{3}^{\perp}= & \left.\begin{array}{rrrr}
1 & 0 & 2 & 0 \\
0 & 1 & 2 & 0 \\
0 & 0 & -1 & 0 \\
0 & 0 & 2 & 1
\end{array}\right], & \mathbf{S}_{4}^{\perp}=\left[\begin{array}{rrrr}
1 & 0 & 0 & 2 \\
0 & 1 & 0 & 2 \\
0 & 0 & 1 & 2 \\
0 & 0 & 0 & -1
\end{array}\right] .
\end{array}
$$

We note that $\mathbf{S}_{i}^{\perp}=\mathbf{S}_{i}^{T}$, the transpose of $\mathbf{S}_{i}$.

By analogy with Theorem 4.3 we might think of an orbit of the dual Apollonian group acting on a single oriented Descartes configuration $\mathcal{D}$, as a "dual Apollonian 
circle packing". It can be viewed algebraically as a set of Descartes configurations or geometrically as a collection of circles in the plane. In the algebraic viewpoint the orbit is a discrete set of points in the parameter space $\mathcal{M}_{\mathbb{D}}$, so is a discrete object. From the geometrical viewpoint, as a collection of circles, which we denote $\mathcal{P}_{\mathcal{D}}^{\perp}$, it has the following weak "packing" property.

Theorem 5.1. No two circles in a dual Apollonian circle packing cross each other. That is, two circles in distinct Descartes configurations in a dual Apollonian circle packing $\mathcal{P}_{\mathcal{D}}^{\perp}$ either coincide, or are tangent to each other, or are disjoint.

We do not give a proof of this theorem here, as it follows from a similar result proved for the super-Apollonian packing in Section 3 of part II. It is also a special case of an $n$-dimensional generalization proved in Section 4.2 of part III.

Figure 9 pictures the circles in a dual packing $\mathcal{P}^{\perp}$. These are circles in Descartes configurations dual to those in the Apollonian packing in Fig. 1. The fractal-like part of

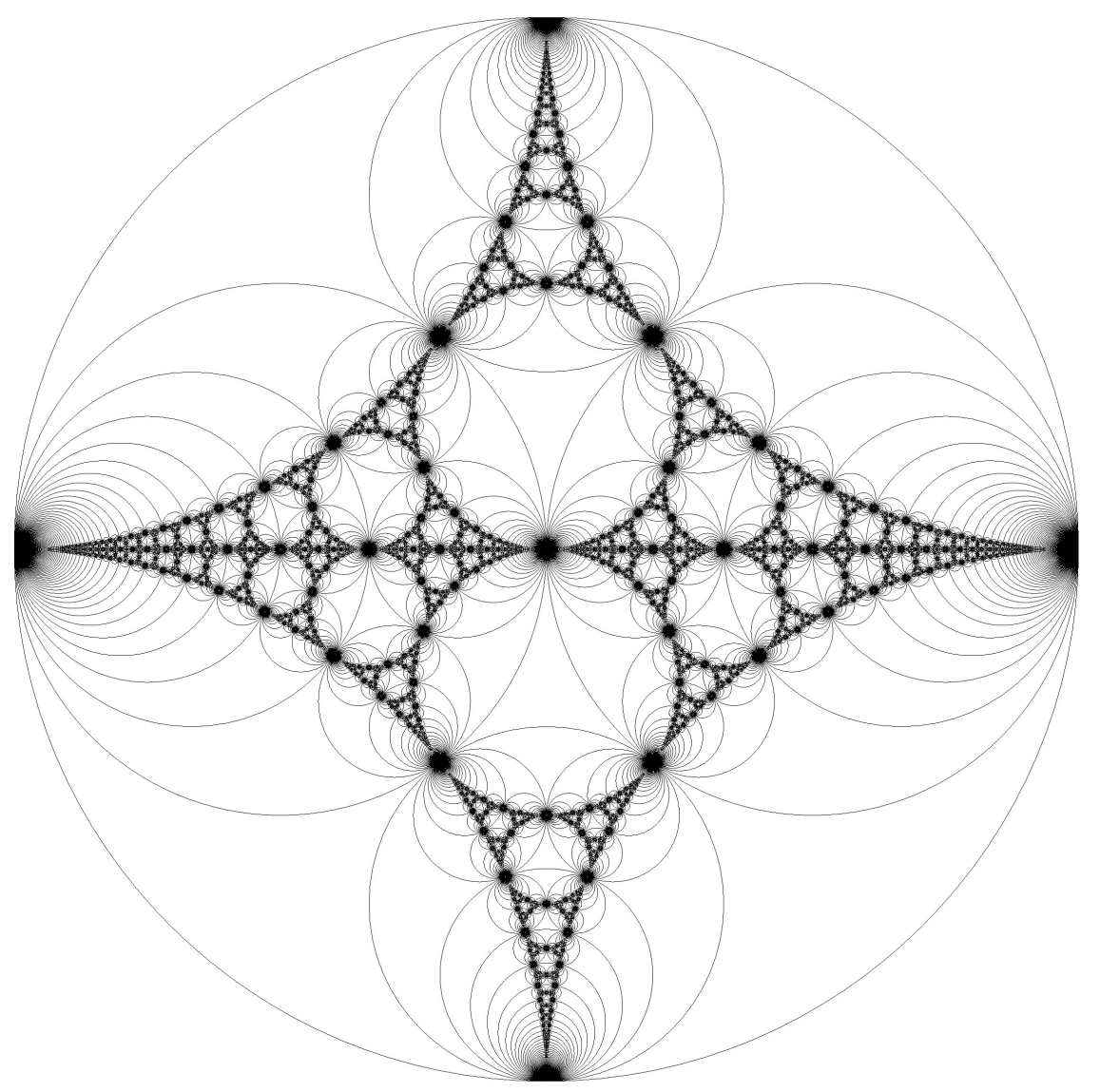

Fig. 9. A dual Apollonian packing. 
this figure is the closure of the set of points at which circles in the dual packing touch. It can be shown that infinitely many circles touch at each such tangency point. Below we show that this fractal set coincides with the limit set of the Apollonian packing generated by the dual Descartes configuration $\mathbf{D}\left(\mathcal{D}_{0}\right)$.

The dual Apollonian group has a simple relation to the Apollonian group.

Theorem 5.2. The dual Apollonian group $\mathcal{A}^{\perp}$ is conjugate to the Apollonian group $\mathcal{A}$ using the duality operator $\mathbf{D} \in \operatorname{Aut}\left(Q_{D}\right)$. This holds at the level of generators, with

$$
\mathbf{D}^{T} \mathbf{S}_{i} \mathbf{D}=\mathbf{S}_{i}^{\perp} \quad \text { for } \quad 1 \leq i \leq 4,
$$

where $\mathbf{D}=\mathbf{D}^{-1}=\mathbf{D}^{T}$.

Proof. This is a straightforward computation.

Theorem 5.3. Given a dual Apollonian packing $\mathcal{P}_{\mathcal{D}_{0}}^{\perp}$ generated by Descartes configuration $\mathcal{D}_{0}$, let $\Sigma$ be the set of all intersection points of the circles in $\mathcal{A}^{\perp}\left(\mathcal{D}_{0}\right)$. Then the closure $\Lambda:=\bar{\Sigma}$ is equal to the residual set $\Lambda\left(\mathcal{P}_{\mathbf{D}\left(\mathcal{D}_{0}\right)}\right)$ of the Apollonian packing $\mathcal{P}_{\mathbf{D}\left(\mathcal{D}_{0}\right)}$ generated by the dual Descartes configuration $\mathbf{D}\left(\mathcal{D}_{0}\right)$.

Proof. Theorem 4.2(3) states that the residual set $\Lambda(\mathcal{P})$ of a geometric Apollonian packing $\mathcal{P}$ is the closure of the set of tangency points $\Sigma^{\prime}$ in $\mathcal{P}$. Thus it will suffice to show that the set of tangency points $\Sigma$ of the circles in the dual packing $\mathcal{P}_{\mathcal{D}_{0}}^{\perp}$ coincides with the set of tangency points $\Sigma^{\prime}$ of circles in the Apollonian packing $\mathcal{P}_{\mathbf{D}\left(\mathcal{D}_{0}\right)}$. Each intersection point of two circles in the dual Apollonian packing $\mathcal{P}_{\mathcal{D}_{0}}^{\perp}$ is an intersection point of circles in some Descartes configuration $\mathcal{D}$ of $\mathcal{A}^{\perp}\left(\mathcal{D}_{0}\right)$. (This follows from the recursive construction of the dual packing.) Each intersection point or circles in $\mathcal{D}$ is an intersection point of circles in the dual Descartes configuration $\mathbf{D}(\mathcal{D})$, which is the configuration having augmented curvature-center coordinates $\mathbf{D W}_{\mathcal{D}}$. Now $\mathbf{D}(\mathcal{D})$ belongs to the Apollonian packing $\mathcal{A}\left(\mathbf{D}\left(\mathcal{D}_{0}\right)\right)$ because

$$
\mathbf{D} \mathbf{W}_{\mathcal{D}}=\mathbf{D}\left(\mathbf{S}_{i_{1}}^{\perp} \cdots \mathbf{S}_{i_{m}}^{\perp} \mathbf{W}_{\mathcal{D}_{0}}\right)=\mathbf{S}_{i_{1}} \cdots \mathbf{S}_{i_{m}}\left(\mathbf{D} \mathbf{W}_{\mathcal{D}_{0}}\right),
$$

using Theorem 5.2. Thus the set $\Sigma$ of intersection points of circles in the dual Apollonian packing $\mathcal{P}_{\mathcal{D}_{0}}^{\perp}$ is contained in the set $\Sigma^{\prime}$ of intersection points of circles in the Apollonian packing $\mathcal{P}_{\mathbf{D}\left(\mathcal{D}_{0}\right)}$. The argument reverses to show the converse is also true: each intersection point of circles in the Apollonian packing $\mathcal{P}_{\mathbf{D}\left(\mathcal{D}_{0}\right)}$ is an intersection point of circles in the dual Apollonian packing $\mathcal{P}_{\mathcal{D}_{0}}^{\perp}$. Thus $\Sigma=\Sigma^{\prime}$.

We have the following dichotomy between the geometric and algebraic views of Apollonian packings and dual Apollonian packings. Viewed geometrically as collections of circles, a dual Apollonian packing $\mathcal{P}^{\perp}$ appears quite different from an Apollonian packing, as the circles are nested to an infinite depth. However, when viewed algebraically as a collection of ordered, oriented Descartes configurations $\mathbb{D}\left(\mathcal{P}^{\perp}\right)$, it is a discrete set given by the orbit of a group $\mathcal{A}^{\perp}$ conjugate to the Apollonian group $\mathcal{S}$, and so is a similar object. 


\section{Super-Apollonian Group}

The Apollonian group $\mathcal{A}$ and dual Apollonian group $\mathcal{A}^{\perp}$ both consist of integer automorphs in $\operatorname{Aut}\left(Q_{D}\right)$. We obtain a larger integral group by combining the two groups, as follows.

Definition 6.1. The super-Apollonian group $\mathcal{A}^{S}$ is the subgroup of $\operatorname{Aut}\left(Q_{D}\right)$ generated by the Apollonian group $\mathcal{A}$ and $\mathcal{A}^{\perp}$ together. We have

$$
\mathcal{A}^{S}=\left\langle\mathbf{S}_{1}, \mathbf{S}_{2}, \mathbf{S}_{3}, \mathbf{S}_{4}, \mathbf{S}_{1}^{\perp}, \mathbf{S}_{2}^{\perp}, \mathbf{S}_{3}^{\perp}, \mathbf{S}_{4}^{\perp}\right\rangle .
$$

The super-Apollonian group $\mathcal{A}^{S}$ is a discrete subgroup of $\operatorname{Aut}\left(Q_{D}\right)$ as a consequence of the fact that all its members are integral matrices.

\subsection{Presentation of Super-Apollonian Group}

We now determine a presentation of the super-Apollonian group, showing that it is a hyperbolic Coxeter group. This result implies that both the Apollonian group and dual Apollonian group are finitely presented, and are hyperbolic Coxeter groups.

Theorem 6.1. The super-Apollonian group

$$
\mathcal{A}^{S}=\left\langle\mathbf{S}_{1}, \mathbf{S}_{2}, \mathbf{S}_{3}, \mathbf{S}_{4}, \mathbf{S}_{1}^{\perp}, \mathbf{S}_{2}^{\perp}, \mathbf{S}_{3}^{\perp}, \mathbf{S}_{4}^{\perp}\right\rangle
$$

is a hyperbolic Coxeter group whose complete set of Coxeter relations are

$$
\begin{aligned}
& \mathbf{S}_{i}^{2}=\left(\mathbf{S}_{i}^{\perp}\right)^{2}=\mathbf{I} \quad \text { for } \quad 1 \leq i \leq 4, \\
& \left(\mathbf{S}_{i} \mathbf{S}_{j}^{\perp}\right)^{2}=\left(\mathbf{S}_{j}^{\perp} \mathbf{S}_{i}\right)^{2}=\mathbf{I} \quad \text { if } \quad i \neq j .
\end{aligned}
$$

Proof. Recall that $\mathbf{S}_{i}^{\perp}=\mathbf{S}_{i}^{T}$. The group $\mathcal{A}^{S}$ satisfies the relations $\mathbf{S}_{i}^{2}=\left(\mathbf{S}_{i}^{T}\right)^{2}=\mathbf{I}$ for $1 \leq i \leq 4$ and it satisfies

$$
\mathbf{S}_{i} \mathbf{S}_{j}^{T}=\mathbf{S}_{j}^{T} \mathbf{S}_{i} \quad \text { for } \quad i \neq j,
$$

which is equivalent to (6.2).

Write words $\mathbf{U}$ in $\mathcal{A}^{S}$ as $\mathbf{U}=\mathbf{U}_{n} \mathbf{U}_{n-1} \cdots \mathbf{U}_{1}$, in which each $\mathbf{U}_{i}$ is a generator $\mathbf{S}_{j}$ or $\mathbf{S}_{j}^{T}$, with the empty word $(n=0)$ being the identity element. We do not need inverses since each generator is its own inverse. A word $\mathbf{U}$ is in normal form if it has the two properties:

(i) $\mathbf{U}_{k} \neq \mathbf{U}_{k-1}$ for $2 \leq k \leq n$.

(ii) If $\mathbf{U}_{k}=\mathbf{S}_{j}^{T}$ for some $j$, then $\mathbf{U}_{k-1} \neq \mathbf{S}_{i}$ for all $i \neq j$.

We can reduce any word to a word in normal form, using the relations to move any symbols $\mathbf{S}_{j}^{T}$ as far to the right in the word as possible. We cancel any adjacent identical 
symbols to make (i) hold. Then move the rightmost $\mathbf{S}_{j}^{T}$ as far to the right as possible, as allowed by (6.3). Repeat the same with the next rightmost symbol $\mathbf{S}_{j}^{T}$. If in the process any adjacent symbols $\left(\mathbf{S}_{j}^{T}\right)^{2}$ occur, cancel them. This process must terminate in a normal form word or the empty word.

The theorem is equivalent to showing that no normal form word with $n \geq 1$ is the identity element in $\mathcal{A}^{S}$. This holds because the reduction to normal form used only the Coxeter relations, so if $\mathcal{A}^{S}$ satisfies an additional nontrivial relation, there would exist some nontrivial normal form word that is the identity.

We proceed by induction on the length $n$ of a word in normal form $\mathbf{U}=\mathbf{U}_{n} \mathbf{U}_{n-1} \cdots \mathbf{U}_{1}$. Note that any suffix $\mathbf{U}_{j-1} \mathbf{U}_{j} \cdots \mathbf{U}_{1}$ of $\mathbf{U}$ is also in normal form. For each $n \geq 1$, let $\mathcal{X}_{n}$ denote the set of all normal form words of length $n$. (By a simple enumeration we can show that the number of normal form words of length $n$ is $9 \cdot 5^{n-1}-1$.) We measure the size of a word $\mathbf{U}$ (viewed as a $4 \times 4$ matrix) as

$$
f(\mathbf{U}):=\mathbf{1}^{T} \mathbf{U} \mathbf{1}=\sum_{i=1}^{4} \sum_{j=1}^{4} \mathbf{U}_{i j}
$$

Thus $f(\mathbf{I})=4$. For $n=1$ we have

$$
f(\mathbf{U})=f\left(\mathbf{U}_{1}\right)=8
$$

in all cases. For $n \geq 2$ if $\mathbf{U} \in \mathcal{X}_{n}$ then $\mathbf{U}^{\prime}=\mathbf{U}_{n-1} \mathbf{U}_{n-2} \cdots \mathbf{U}_{1} \in \mathcal{X}_{n-1}$, and we will prove

$$
f(\mathbf{U})>f\left(\mathbf{U}^{\prime}\right) .
$$

If so, then

$$
f(\mathbf{U}) \geq f\left(\mathbf{U}_{1}\right) \geq 8,
$$

hence $\mathbf{U} \neq \mathbf{I}$, which will complete the proof.

We let

$$
\mathbf{r}(\mathbf{U}):=\left(r_{1}(\mathbf{U}), r_{2}(\mathbf{U}), r_{3}(\mathbf{U}), r_{4}(\mathbf{U})\right)^{T}=\mathbf{U} \mathbf{1}
$$

be the vector of row sums of $\mathbf{U}$. Now

$$
\begin{aligned}
& f\left(\mathbf{S}_{1} \mathbf{U}^{\prime}\right)=\mathbf{1}^{T} \mathbf{S}_{1} \mathbf{r}\left(\mathbf{U}^{\prime}\right)=(-1,3,3,3) \mathbf{r}\left(\mathbf{U}^{\prime}\right)=-4 r_{1}\left(\mathbf{U}^{\prime}\right)+3 f\left(\mathbf{U}^{\prime}\right), \\
& f\left(\mathbf{S}_{1}^{T} \mathbf{U}^{\prime}\right)=\mathbf{1}^{T} \mathbf{S}_{1}^{T} \mathbf{r}\left(\mathbf{U}^{\prime}\right)=(5,1,1,1) \mathbf{r}(\mathbf{U})=4 r_{1}\left(\mathbf{U}^{\prime}\right)+f\left(\mathbf{U}^{\prime}\right)
\end{aligned}
$$

with similar formulas in the other cases. To prove (6.6) it is therefore sufficient to prove the following two assertions, for $1 \leq h \leq 4$.

(1) If $\mathbf{U}_{n}=\mathbf{S}_{h}$, so that $\mathbf{U}_{n-1} \neq \mathbf{S}_{h}$, then $2 r_{h}\left(\mathbf{U}^{\prime}\right)<f\left(\mathbf{U}^{\prime}\right)$.

(2) If $\mathbf{U}_{n}=\mathbf{S}_{h}^{T}$, so that $U_{n-1} \neq \mathbf{S}_{h}^{T}$ and $U_{n-1} \neq \mathbf{S}_{i}$ for $i \neq h$, then $r_{h}\left(\mathbf{U}^{\prime}\right)>0$.

Instead of proving (1) and (2), we prove by induction on $n$ the following three assertions for all $\mathbf{U} \in \mathcal{X}_{n}$. Here $(h, i, j, k)$ always denotes some permutation of $(1,2,3,4)$ in 
what follows.

(i) For $1 \leq i, j \leq 4$, with $i \neq j$,

$$
r_{i}(\mathbf{U})+r_{j}(\mathbf{U})>0 .
$$

(ii) If $\mathbf{U}_{n}=\mathbf{S}_{h}$, then

$$
\begin{aligned}
r_{h}(\mathbf{U}) & >0, \\
r_{i}(\mathbf{U}) & <r_{h}(\mathbf{U})+r_{j}(\mathbf{U})+r_{k}(\mathbf{U}), \\
r_{j}(\mathbf{U}) & <r_{h}(\mathbf{U})+r_{i}(\mathbf{U})+r_{k}(\mathbf{U}), \\
r_{k}(\mathbf{U}) & <r_{h}(\mathbf{U})+r_{i}(\mathbf{U})+r_{j}(\mathbf{U}) .
\end{aligned}
$$

(iii) If $\mathbf{U}_{n}=\mathbf{S}_{h}^{T}$ then

$$
\begin{aligned}
r_{h}(\mathbf{U}) & <0, \\
r_{i}(\mathbf{U}) & <r_{h}(\mathbf{U})+r_{j}(\mathbf{U})+r_{k}(\mathbf{U}), \\
r_{j}(\mathbf{U}) & <r_{h}(\mathbf{U})+r_{i}(\mathbf{U})+r_{k}(\mathbf{U}), \\
r_{k}(\mathbf{U}) & <r_{h}(\mathbf{U})+r_{i}(\mathbf{U})+r_{j}(\mathbf{U}) .
\end{aligned}
$$

Note that (i) implies that at most one of the row sums of $\mathbf{U}$ can be negative. If proved, (i)-(iii) imply (1) and (2), which themselves imply (6.6), completing the proof of the theorem.

The induction hypotheses (i)-(iii) holds for $n=1$, since $r\left(\mathbf{S}_{h}\right)$ is a permutation of $(5,1,1,1)^{T}$ and $r\left(\mathbf{S}_{h}^{T}\right)$ is a permutation of $(-1,3,3,3)^{T}$. Suppose it is true for $n$. For the induction step, write $\mathbf{U} \in \mathcal{X}_{n+1}$ as $\mathbf{U}=\mathbf{U}_{n+1} \mathbf{U}^{\prime}$ with $\mathbf{U}^{\prime} \in \mathcal{X}_{n}$ and abbreviate $r_{i}^{\prime}=r_{i}\left(\mathbf{U}^{\prime}\right)$.

Case 1: $U_{n+1}=\mathbf{S}_{h}$ for some $h$. We must verify (i) and (ii) for $\mathbf{U}$. We have

$$
\begin{aligned}
& r_{h}(\mathbf{U})=r_{h}\left(\mathbf{S}_{h} \mathbf{U}^{\prime}\right)=-r_{h}^{\prime}+2 r_{i}^{\prime}+2 r_{j}^{\prime}+2 r_{k}^{\prime}, \\
& r_{i}(\mathbf{U})=r_{i}\left(\mathbf{S}_{h} \mathbf{U}^{\prime}\right)=r_{i}^{\prime}, \\
& r_{j}(\mathbf{U})=r_{j}\left(\mathbf{S}_{h} \mathbf{U}^{\prime}\right)=r_{j}^{\prime}, \\
& r_{k}(\mathbf{U})=r_{k}\left(\mathbf{S}_{h} \mathbf{U}^{\prime}\right)=r_{k}^{\prime} .
\end{aligned}
$$

To verify (i), all cases not involving $r_{h}(\mathbf{U})$ follow from the induction hypothesis. To show $r_{h}(\mathbf{U})+r_{i}(\mathbf{U})>0$ note that

$$
r_{h}(\mathbf{U})+r_{i}(\mathbf{U})=-r_{h}^{\prime}+3 r_{i}^{\prime}+2 r_{j}^{\prime}+2 r_{k}^{\prime} .
$$

Now $\mathbf{U}_{n} \neq \mathbf{S}_{h}$ since $\mathbf{U} \in \mathcal{X}_{n+1}$, and for all cases except $\mathbf{U}_{n}=\mathbf{S}_{h}^{T}$ the induction hypotheses (ii) and (iii) give $r_{h}^{\prime}<r_{i}^{\prime}+r_{j}^{\prime}+r_{k}^{\prime}$, whence

$$
r_{h}(\mathbf{U})+r_{i}(\mathbf{U}) \geq 2 r_{i}^{\prime}+r_{j}^{\prime}+r_{k}^{\prime}=\left(r_{i}^{\prime}+r_{j}^{\prime}\right)+\left(r_{i}^{\prime}+r_{k}^{\prime}\right)>0,
$$

using induction hypothesis (i). If $\mathbf{U}_{n}=\mathbf{S}_{h}^{T}$, then by hypothesis (iii) $r_{h}^{\prime}<0$, hence $r_{i}^{\prime}, r_{j}^{\prime}, r_{k}^{\prime}>0$ by hypothesis (i), so all terms on the right side of (6.8) are positive, so 
$r_{h}(\mathbf{U})+r_{i}(\mathbf{U})>0$. The cases $r_{h}(\mathbf{U})+r_{j}(\mathbf{U})>0$ and $r_{h}(\mathbf{U})+r_{k}(\mathbf{U})>0$ follow similarly. To verify (ii) for $\mathbf{U}$ it suffices to prove

$$
\begin{aligned}
0 & <-r_{h}^{\prime}+2 r_{i}^{\prime}+2 r_{j}^{\prime}+2 r_{k}^{\prime}, \\
r_{i}^{\prime} & <-r_{h}^{\prime}+2 r_{i}^{\prime}+3 r_{j}^{\prime}+3 r_{k}^{\prime}, \\
r_{j}^{\prime} & <-r_{h}^{\prime}+3 r_{i}^{\prime}+2 r_{j}^{\prime}+3 r_{k}^{\prime}, \\
r_{k}^{\prime} & <-r_{h}^{\prime}+3 r_{i}^{\prime}+3 r_{j}^{\prime}+2 r_{k}^{\prime},
\end{aligned}
$$

which is equivalent to

$$
\begin{aligned}
r_{h}^{\prime} & <2 r_{i}^{\prime}+2 r_{j}^{\prime}+2 r_{k}^{\prime}, \\
r_{h}^{\prime} & <r_{i}^{\prime}+3 r_{j}^{\prime}+3 r_{k}^{\prime}, \\
r_{h}^{\prime} & <3 r_{i}^{\prime}+r_{j}^{\prime}+3 r_{k}^{\prime}, \\
r_{k}^{\prime} & <3 r_{i}^{\prime}+3 r_{j}+r_{k}^{\prime} .
\end{aligned}
$$

There are three cases, according as $\mathbf{U}_{n}=\mathbf{S}_{i}, \mathbf{U}_{n}=\mathbf{S}_{i}^{T}$ with $i \neq h$ and $\mathbf{U}_{n}=\mathbf{S}_{h}^{T}$. In the first two of these, the induction hypotheses give

$$
r_{h}^{\prime}<r_{i}^{\prime}+r_{j}^{\prime}+r_{k}^{\prime}
$$

which with induction hypothesis (i) immediately yields (6.9). If $\mathbf{U}_{n}=\mathbf{S}_{h}^{T}$ then by (iii), $r_{h}^{\prime}<0$, whence $r_{i}^{\prime}, r_{j}^{\prime}, r_{k}^{\prime}>0$ by (i) so (6.9) is immediate. This finishes Case 1.

Case 2: $\mathbf{U}_{n+1}=\mathbf{S}_{h}^{T}$ for some $h$. We must verify (i) and (iii). We have

$$
\begin{aligned}
r_{h}(\mathbf{U}) & =r_{h}\left(\mathbf{S}_{h}^{T} \mathbf{U}^{\prime}\right)=-r_{h}^{\prime}, \\
r_{i}(\mathbf{U}) & =r_{i}\left(\mathbf{S}_{h}^{T} \mathbf{U}^{\prime}\right)=2 r_{h}^{\prime}+r_{i}^{\prime}, \\
r_{j}(\mathbf{U}) & =r_{j}\left(\mathbf{S}_{h}^{T} \mathbf{U}^{\prime}\right)=2 r_{h}^{\prime}+r_{j}^{\prime}, \\
r_{k}(\mathbf{U}) & =r_{k}\left(\mathbf{S}_{h}^{T} \mathbf{U}^{\prime}\right)=2 r_{h}^{\prime}+r_{j}^{\prime} .
\end{aligned}
$$

To prove (i), note first that

$$
r_{h}(\mathbf{U})+r_{i}(\mathbf{U})=r_{h}^{\prime}+r_{i}^{\prime}>0,
$$

using (i) for $\mathbf{U}^{\prime}$, and similar inequalities hold for other cases involving $r_{h}(\mathbf{U})$. The remaining cases are all of the form $r_{i}(\mathbf{U})+r_{j}(\mathbf{U})>0$ with $i, j \neq h$, and the proofs for each are similar. We have

$$
r_{i}(\mathbf{U})+r_{j}(\mathbf{U})=4 r_{h}^{\prime}+r_{i}^{\prime}+r_{j}^{\prime}=2 r_{h}^{\prime}+\left(r_{h}^{\prime}+r_{i}^{\prime}\right)+\left(r_{h}^{\prime}+r_{j}^{\prime}\right)>2 r_{h}^{\prime},
$$

using induction hypothesis (i). We now show $r_{h}^{\prime}>0$ holds in all cases. Since $\mathbf{U} \in \mathcal{X}_{n+1}$, we have $\mathbf{U}_{n} \neq \mathbf{S}_{h}^{T}$ and $\mathbf{U}_{n} \neq \mathbf{S}_{i}$ with $i \neq h$. If $\mathbf{U}_{n}=\mathbf{S}_{j}^{T}$ for some $j \neq h$ then hypothesis (iii) for $\mathbf{U}^{\prime}$ says that $r_{j}^{\prime}<0$, whence we must have $r_{h}^{\prime}>0$. If $\mathbf{U}_{n}=\mathbf{S}_{h}$ then hypothesis 
(ii) for $\mathbf{U}^{\prime}$ says $r_{h}^{\prime}>0$. Thus $r_{h}^{\prime}>0$ in all cases, and (i) holds for $\mathbf{U}$. To prove (ii) we must prove

$$
\begin{aligned}
r_{h}^{\prime} & <0, \\
2 r_{h}^{\prime}+r_{i}^{\prime} & <3 r_{h}^{\prime}+r_{j}^{\prime}+r_{k}^{\prime}, \\
2 r_{h}^{\prime}+r_{j}^{\prime} & <3 r_{h}^{\prime}+r_{i}^{\prime}+r_{k}^{\prime}, \\
2 r_{h}^{\prime}+r_{k}^{\prime} & <3 r_{h}^{\prime}+r_{i}^{\prime}+r_{j}^{\prime},
\end{aligned}
$$

which is equivalent to

$$
\begin{aligned}
r_{h}^{\prime} & >0, \\
r_{i}^{\prime} & <r_{h}^{\prime}+r_{j}^{\prime}+r_{k}^{\prime}, \\
r_{j}^{\prime} & <r_{h}^{\prime}+r_{i}^{\prime}+r_{k}^{\prime}, \\
r_{k}^{\prime} & <r_{h}^{\prime}+r_{i}^{\prime}+r_{j}^{\prime} .
\end{aligned}
$$

We verified $r_{h}^{\prime}>0$ already. For the remainder there are two cases, according as $\mathbf{U}_{n}=\mathbf{S}_{h}$ or $\mathbf{U}_{n}=\mathbf{S}_{i}^{T}$ for some $i \neq h$. If $\mathbf{U}_{n}=\mathbf{S}_{h}$ the three remaining inequalities in (6.10) follow from inductive hypothesis (ii) for $\mathbf{U}^{\prime}$. If $\mathbf{U}_{n}=\mathbf{S}_{i}^{T}$ the inductive hypothesis (iii) gives the last two inequalities in (6.10), and also that $r_{i}^{\prime}<0$. By hypothesis (i) for $\mathbf{U}^{\prime}$ this implies $r_{j}^{\prime}, r_{h}^{\prime}, r_{k}^{\prime}>0$ hence $r_{i}^{\prime}<0<r_{h}^{\prime}+r_{j}^{\prime}+r_{k}^{\prime}$ which verifies (6.10) in this case, and finishes Case 2.

The induction is complete.

\subsection{Apollonian Super-Packings}

We now define an Apollonian super-packing to be a set of (ordered, oriented) Descartes configurations forming an orbit of the super-Apollonian group $\mathcal{A}^{S}$, acting on a single such configuration.

The study of Apollonian super-packings forms the subject matter of part II. We call the set of circles in all the Descartes configurations in such a super-packing a geometric Apollonian super-packing. These circles comprise four orbits of a group of Möbius transformations

$$
\mathcal{G}_{\mathcal{A}^{s}}(\mathcal{D})=\left\langle\mathfrak{s}_{1}, \mathfrak{s}_{2}, \mathfrak{s}_{3}, \mathbf{s}_{4}, \mathbf{s}_{1}^{\perp}, \mathbf{s}_{2}^{\perp}, \mathbf{s}_{3}^{\perp}, b s_{4}^{\perp}\right\rangle,
$$

where $\mathfrak{s}_{i}$ and $\mathfrak{s}_{i}^{\perp}$ were defined in Section 3.3. In terms of the space $\mathcal{M}_{\mathbb{D}}$ of Descartes configurations this group action is given by $\tilde{\mathcal{G}}_{\mathcal{A}^{s}}(\mathcal{D}) \subset \operatorname{Aut}\left(Q_{W}\right)$ defined by

$$
\tilde{\mathcal{G}}_{\mathcal{A}^{s}}(\mathcal{D}):=\mathbf{W}_{\mathcal{D}}^{-1} \mathcal{A}^{S} \mathbf{W}_{\mathcal{D}},
$$

using the isomorphism $\operatorname{Aut}\left(Q_{W}\right)=\operatorname{Möb}(2) \times\{\mathbf{I},-\mathbf{I}\}$ given in Theorem A.2 of Appendix A.

In part II we show that the individual circles in a geometric super-packing form a "packing" in the weak sense that no two circles cross each other, although circles can be nested. This is a remarkable geometric fact, because we also show in part II that a strongly integral Apollonian super-packing necessarily contains a copy of every integral Apollonian circle packing, with all these copies (essentially) contained inside the square $0 \leq x \leq 2,0 \leq y \leq 2$. All these Apollonian packings fit together in such a way that all the circles in these packings manage not to cross each other. 


\section{Acknowledgments}

The authors thank Ludwig Balke for suggesting that the two group actions in Section 3 should correspond to a left action and a right action. The authors are grateful for helpful comments from Andrew Odlyzko, Eric Rains, Jim Reeds and Neil Sloane. The authors thank the reviewer for incisive comments leading to reorganization of the paper.

\section{Appendix A. Möbius Group Action}

The (general) Möbius group Möb(2) is the group of Möbius transformations, allowing reflections (including complex conjugation). This group is denoted $G M\left(\hat{\mathbb{R}}^{2}\right)$ in p. 23 in [3], and is also known as the conformal group. The group $\operatorname{Möb}(2)_{+} \simeq \operatorname{PSL}(2, \mathbb{C})=$ $S L(2, \mathbb{C}) /\{ \pm \mathbf{I}\}$ consists of the orientation-preserving maps of $\hat{\mathbb{C}}=\mathbb{R}^{2} \cup\{\infty\}$. The group $M o ̈ b(2)$ is a six-dimensional real Lie group which has two connected components corresponding to orientation-preserving ${ }^{4}$ and orientation-reversing transformations. It can be written as a semi-direct product

$$
\operatorname{Möb}(2) \cong \operatorname{Möb}(2)_{+} \rtimes\{1, \mathfrak{c}\},
$$

in which $\mathfrak{c}$ denotes complex conjugation. Möbius transformations take circles to circles (or straight lines) and preserve angles. Thus they take ordered Descartes configurations to ordered Descartes configurations. Concerning orientation, we show that although Möbius transformations can reverse orientation of a single circle, they preserve (total) orientation of oriented Descartes configurations. Recall that the total orientation of an oriented Descartes configuration is the sign of the sum of the (signed) curvatures of the circles in it.

Theorem A.1. Möbius transformations preserve the total orientation of oriented Descartes configurations.

Proof. Positively oriented Descartes configurations are characterized by the four circles having disjoint "interiors," specified by the positive orientation. Möbius transformations take Descartes configurations to Descartes configurations, and preserve the "disjoint interior" property, hence preserve positive orientation, as given by normal vectors to the circle. The result for negatively oriented Descartes configurations holds since all normal vectors are reversed from the positively oriented case.

We now consider a group $G M^{*}(2)$ with four connected components, which we term the extended General Möbius group, defined by $G M^{*}(2):=\operatorname{Möb}(2) \times\{\mathbf{I},-\mathbf{I}\}$. Here $\{\mathbf{I},-\mathbf{I}\}$ are in the center of this group, and we write elements of $G M^{*}(2)$ as $\pm \mathfrak{g}$, in which $\mathfrak{g} \in \operatorname{Möb}(2)$, and the sign indicates which of $\pm \mathbf{I}$ occurs. We have

$$
G M^{*}(2) \cong S L(2, \mathbb{C}) \rtimes\{\mathbf{I}, \mathfrak{c}\}
$$

\footnotetext{
${ }^{4}$ The terminology "orientation" here refers to the invariant $\operatorname{det}(\mathbf{M})$.
} 
Table 1. Group Isomorphisms

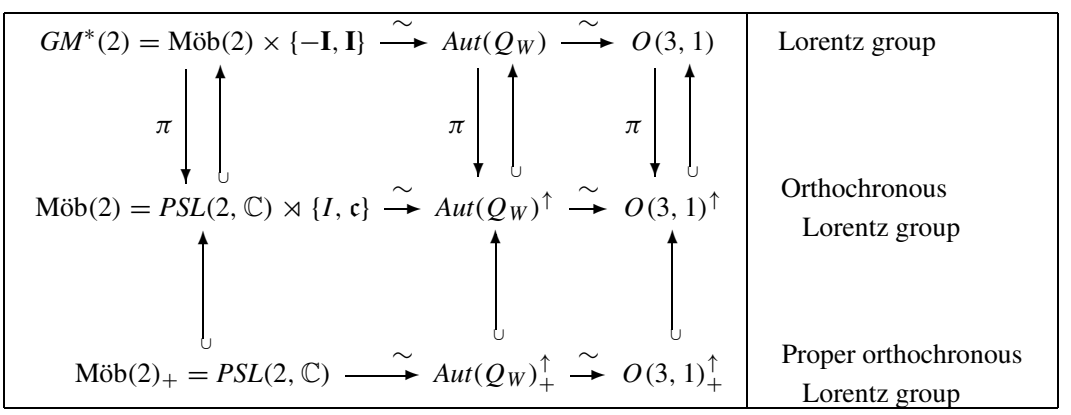

The main object of this Appendix is to define an action of $G M^{*}(2)$ on the right on the parameter space $\mathcal{M}_{\mathbb{D}}$, given in the next theorem. This amounts to finding an explicit isomorphism between $G M^{*}(2)$ and $\operatorname{Aut}\left(Q_{W}\right)$, which appears as the horizontal arrow on the left in the top row in Table 1. This map when restricted to the smaller groups Möb(2) and $\mathrm{Möb}(2)_{+}$give the other two horizontal isomorphisms on the left side of the table. Table 1 also indicates isomorphisms on its right side to the Lorentz group $O(3,1)$ and corresponding subgroups, which we defer discussing until after the following result.

Theorem A.2. Let $G M^{*}(2):=\operatorname{Möb}(2) \times\{\mathbf{I},-\mathbf{I}\}$. There is a unique isomorphism $\pi: G M^{*}(2) \rightarrow \operatorname{Aut}\left(Q_{W}\right)$, with image elements $\mathbf{V}_{ \pm \mathfrak{g}}:=\pi( \pm \mathfrak{g})$, such that the following hold:

(i) For $\mathfrak{g} \in \mathrm{Möb}(2)$ the augmented curvature-center coordinates for each ordered, oriented Descartes configuration $\mathcal{D}$ satisfy

$$
\mathbf{W}_{\mathfrak{g}(\mathcal{D})}=\mathbf{W}_{\mathcal{D}} \mathbf{V}_{\mathfrak{g}}^{-1} .
$$

(ii) The action of -I on augmented curvature-center coordinates is

$$
\mathbf{W}_{-\mathcal{D}}=\mathbf{W}_{\mathcal{D}} \mathbf{V}_{-\mathbf{I}}^{-1}=-\mathbf{W}_{\mathcal{D}}
$$

Proof. We compute the action of Möb(2) acting on augmented curvature-center coordinates. Let $\left(\bar{b}, b, w_{1}, w_{2}\right)=\left(\left(x_{1}^{2}+y_{1}^{2}-r^{2}\right) / r, 1 / r, x_{1} / r, y_{1} / r\right)$ be the augmented curvature-center coordinates of the circle

$$
\left(x-x_{1}\right)^{2}+\left(y-y_{1}\right)^{2}=r^{2} .
$$

This circle can be recovered from these coordinates via

$$
\left(b x-w_{1}\right)^{2}+\left(b y-w_{2}\right)^{2}=1,
$$

and the orientation of the circle (inside versus outside) is determined by the sign of $b$. An oriented "circle at infinity" is a line given by

$$
x \cos \theta+y \sin \theta=m,
$$


and its associated curvature-center coordinates are

$$
\left(\bar{b}, b, w_{1}, w_{2}\right)=(2 m, 0, \cos \theta, \sin \theta) .
$$

Here the orientation is given by the convention that the normal $(\cos \theta, \sin \theta)$ points inward.

The group Möb(2) is generated by

(1) translations $\mathfrak{t}_{z_{0}}=z+z_{0}$;

(2) dilations $\mathfrak{d}_{\lambda}(z)=\lambda z$ with $\lambda \in \mathbb{C}, \lambda \neq 0$;

(3) the conjugation $\mathfrak{c}(z)=\bar{z}$;

(4) the inversion in the unit circle $j(z)=1 / \bar{z}=z /|z|^{2}$.

Given $\mathfrak{g} \in \operatorname{Möb}(2)$, we let $\tilde{\mathfrak{g}}$ denote the corresponding action on the curvature-center coordinates of an oriented circle. The action of translation by $z_{0}=x_{0}+i y_{0}$ is

$$
\begin{aligned}
\tilde{\mathfrak{t}}_{z 0}(\bar{b}, b, & \left.w_{1}, w_{2}\right) \\
& =\left(\bar{b}+2 w_{1} x_{0}+2 w_{2} y_{0}+b\left(x_{0}^{2}+y_{0}^{2}\right), b, w_{1}+b x_{0}, w_{2}+b y_{0}\right) .
\end{aligned}
$$

The action of a dilation with $\lambda=r e^{i \theta}(r>0)$ is given by

$$
\tilde{\mathfrak{d}}_{\lambda}\left(\bar{b}, b, w_{1}, w_{2}\right)=\left(r \bar{b}, b / r, w_{1} \cos \theta-w_{2} \sin \theta, w_{1} \sin \theta+w_{2} \cos \theta\right) .
$$

The action of complex conjugation is

$$
\tilde{\mathfrak{c}}\left(\bar{b}, b, w_{1}, w_{2}\right)=\left(\bar{b}, b, w_{1},-w_{2}\right) .
$$

The action of inversion is

$$
\tilde{\mathfrak{j}}\left(\bar{b}, b, w_{1}, w_{2}\right)=\left(b, \bar{b}, w_{1}, w_{2}\right) .
$$

All of these actions apply to "circles at infinity" and extend to linear maps on the $4 \times 4$ matrices $\mathbf{W}_{\mathcal{D}}$.

The translation operation is given by right multiplication by the matrix

$$
\mathbf{V}_{\mathfrak{t}_{z_{0}}}^{-1}:=\left[\begin{array}{ccrc}
1 & 0 & 0 & 0 \\
x_{0}^{2}+y_{0}^{2} & 1 & x_{0} & y_{0} \\
2 x_{0} & 0 & 1 & 0 \\
2 y_{0} & 0 & 0 & 1
\end{array}\right],
$$

and one verifies (A.1) holds by direct computation.

For the dilation $\mathfrak{d}_{\lambda}$, with $\lambda=r e^{i \theta}(r>0)$ the right action is by the matrix

$$
\mathbf{V}_{\mathfrak{d}_{\lambda}}^{-1}:=\left[\begin{array}{cccc}
r & 0 & 0 & 0 \\
0 & 1 / r & 0 & 0 \\
0 & 0 & \cos \theta & \sin \theta \\
0 & 0 & -\sin \theta & \cos \theta
\end{array}\right] .
$$

For complex conjugation $\mathfrak{c}$, the right action is by the matrix

$$
\mathbf{V}_{\mathfrak{c}}^{-1}=\mathbf{V}_{\mathfrak{c}}:=\left[\begin{array}{rrrr}
1 & 0 & 0 & 0 \\
0 & 1 & 0 & 0 \\
0 & 0 & 1 & 0 \\
0 & 0 & 0 & -1
\end{array}\right] .
$$


For the inversion $\mathfrak{j}_{C}$ in the unit circle, the permutation matrix

$$
\mathbf{V}_{\mathfrak{j}_{C}}^{-1}=\mathbf{V}_{\mathfrak{j}_{C}}=P_{(12)}=\left[\begin{array}{cccc}
0 & 1 & 0 & 0 \\
1 & 0 & 0 & 0 \\
0 & 0 & 1 & 0 \\
0 & 0 & 0 & 1
\end{array}\right] \text {. }
$$

It is easy to verify that the above matrices are all in $\operatorname{Aut}\left(Q_{W}\right)^{\uparrow}$, so that the map so far defines a homomorphism of $\operatorname{Möb}(2)$ into $\operatorname{Aut}\left(Q_{W}\right)^{\uparrow} \simeq O(3,1)^{\uparrow}$, identified with the isochronous Lorentz group. The group Möb(2) acts simply transitively on ordered Descartes configurations, as observed by Wilker [42, Theorem 3, p. 394], and the group $\operatorname{Aut}\left(Q_{W}\right)$ acts simply transitively on ordered, oriented Descartes configurations by Theorem 3.3. Because $\operatorname{Aut}\left(Q_{W}\right)^{\uparrow}$ is of index 2 in $\operatorname{Aut}\left(Q_{W}\right) \simeq O(3,1)$, we conclude that the map so far defines an isomorphism of $\operatorname{Möb}(2)$ onto $\operatorname{Aut}\left(Q_{W}\right)^{\uparrow}$.

To complete the proof, we define the action of $-\mathbf{I}$ to be

$$
\left(\mathbf{V}_{-\mathbf{I}}\right)^{-1}=\mathbf{V}_{-\mathbf{I}}=-\mathbf{I} .
$$

It has the effect of reversing (total) orientation of the Descartes configuration, and does not correspond to a conformal transformation. Since $-\mathbf{I} \notin \operatorname{Aut}\left(Q_{W}\right)^{\uparrow}$, adding it gives the desired isomorphism of $G M(2)$ onto $\operatorname{Aut}\left(Q_{W}\right)$.

In terms of the natural coordinates on $\operatorname{Möb}(2)_{+} \simeq \operatorname{PSL}(2, \mathbb{C})$ the homomorphism $\pi(\cdot)$ given in Theorem A.2 is a nonlinear map. This can be clearly seen in (A.10), where both $\mathbf{U}_{\mathfrak{d}_{\lambda}}$ and $\mathbf{U}_{\mathfrak{d}_{\lambda}}^{-1}$ are nonlinear functions of the coordinates of

$$
\mathfrak{d}_{\lambda}=\left[\begin{array}{cc}
\sqrt{\lambda} & 0 \\
0 & 1 / \sqrt{\lambda}
\end{array}\right] \in S L(2, \mathbb{C})
$$

Here the two choices of $\pm \sqrt{\lambda}$ give a well defined matrix modulo $\pm I$.

We now return to the data in Table 1 giving the isomorphisms of $A u t\left(Q_{W}\right)$ and its subgroups to the Lorentz group $O(3,1)$ and its two subgroups $O(3,1)^{\uparrow}$ the orthochronous Lorentz group, and $O(3,1)_{+}^{\uparrow}$, the proper orthochronous Lorentz group, using the terminology of Wilker [42]. An orthochronous Lorentz transformation is one that preserves the arrow of time; Table 1 shows this corresponds to preserving total orientation of a Descartes configuration. The set of isomorphisms given by the three horizontal arrows on the right in Table 1 are obtained by any fixed choice of matrix $\mathbf{A}$ that intertwines $Q_{W}$ and $Q_{\mathcal{L}}$ by $\mathbf{Q}_{W}=\mathbf{A}^{T} \mathbf{Q}_{\mathcal{L}} \mathbf{A}$, in which case the isomorphism is $\operatorname{Aut}\left(Q_{W}\right)=\mathbf{A}^{-1} O(3,1) \mathbf{A}$ sending $\mathbf{V} \mapsto \mathbf{A V A}^{-1}$. To preserve the underlying rational structure of these forms, one must choose $\mathbf{A}$ to be a rational matrix, for example the integral matrix given in (3.17).

There is a different choice of intertwining matrix, for the rightmost horizontal rows in Table 1, which will allow us to make the composed horizontal maps in Table 1 from the Möbius group to the Lorentz groups compatible with the framework used by Wilker [42]. In Theorem 10 in [42] Wilker gave an explicit isomorphism of $\operatorname{PSL}(2, \mathbb{C})$ onto the 
proper orthochronous Lorentz group $O(3,1)_{+}^{\uparrow}$. It maps ${ }^{5}$

$$
\pm\left[\begin{array}{ll}
a & b \\
c & d
\end{array}\right] \longmapsto\left[\begin{array}{cccc}
\frac{1}{2}\left(|a|^{2}+|b|^{2}\right. & \Im(a \bar{c}+b \bar{d}) & \frac{1}{2}\left(|a|^{2}+|b|^{2}\right. & \Re(a \bar{c}+b \bar{d}) \\
\left.+|c|^{2}+|d|^{2}\right) & & \left.-|c|^{2}-|d|^{2}\right) & \\
\Im(-a \bar{b}-c \bar{d}) & \Re(a \bar{d}-b \bar{c}) & \Im(-a \bar{b}+c \bar{d}) & \Im(-a \bar{d}+b \bar{c}) \\
\frac{1}{2}\left(|a|^{2}-|b|^{2}\right. & \Im(a \bar{c}-b \bar{d}) & \frac{1}{2}\left(|a|^{2}-|b|^{2}\right. & \Re(a \bar{c}-b \bar{d}) \\
\left.+|c|^{2}-|d|^{2}\right) & & \left.-|c|^{2}+|d|^{2}\right) & \\
\Re(a \bar{b}+c \bar{d}) & \Im(a \bar{d}+b \bar{c}) & \Re(a \bar{b}-c \bar{d}) & \Re(a \bar{d}+b \bar{c})
\end{array}\right] .
$$

One can identify this map with the composed map in the last row of Table 1 from the first column to the last column, if one chooses the map $\operatorname{Aut}\left(Q_{W}\right) \rightarrow \operatorname{Aut}\left(Q_{\mathcal{L}}\right)$ to be $\mathbf{V} \mapsto \mathbf{Z V Z}^{-1}$ for a particular intertwining matrix $\mathbf{Z}$, which is unique up to multiplication by $\pm \mathbf{I}$. It has irrational entries, and is

$$
\mathbf{Z}:= \pm \sqrt{2}\left[\begin{array}{rrrr}
1 & 1 & 0 & 0 \\
0 & 0 & 0 & -1 \\
1 & -1 & 0 & 0 \\
0 & 0 & -1 & 0
\end{array}\right] .
$$

\section{Appendix B. Groups in Hyperbolic 3-Space Associated to Apollonian Packings}

In this appendix we describe two different discrete groups of isometries of hyperbolic 3-space $\mathbb{H}^{3}$ associated to an Apollonian packing. We specify these groups as groups of Möbius transformations of $\hat{\mathbb{C}}$.

Any Möbius transformation of $\hat{\mathbb{C}}=\hat{\mathbb{R}}^{2}$ has a unique Poincaré lift to an isometry of hyperbolic 3-space $\mathbb{H}^{3}$, viewed as the upper half-space of $\hat{\mathbb{R}}^{3}=\mathbb{R}^{3} \cup\{\infty\}$, as described in Section 3.3 of [3]. Here $\hat{\mathbb{C}}$ is identified with the ideal boundary of $\hat{\mathbb{R}}^{3}$. In this way any group of Möbius transformations acting on $\hat{\mathbb{C}}$ lifts to a group of hyperbolic isometries.

A classical Schottky group is a group $\Gamma=\left\langle\mathfrak{g}_{1}, \ldots, \mathfrak{g}_{n}\right\rangle$ of Möbius transformations associated to $2 n$ circles in the plane $\hat{\mathbb{C}}$ having disjoint interiors, but possibly touching on their boundaries. The group has $n$ generators $\mathfrak{g}_{j}$, in which the $j$ th generator of the group maps the exterior of circle $C_{2 j-1}$ onto the interior of circle $C_{2 j}$. Schottky groups consist entirely of holomorphic Möbius transformations. (Note: knowing a single $\mathfrak{g}_{j}$ alone does not determine the circles $C_{2 j-1}$ and $C_{2 j}$ uniquely.)

Mumford et al. [33, Chapter 7] observe that given an Apollonian circle packing $\mathcal{P}_{\mathcal{D}}$ generated by a Descartes configuration $\mathcal{D}$, there is a Schottky group $\Gamma(\mathcal{D})$ in hyperbolic 3-space $\mathbb{H}^{3}$ whose limit set is exactly the limit set $\Lambda_{\mathcal{D}}$ of the Apollonian packing $\mathcal{P}_{\mathcal{D}}$. The Schottky group $\Gamma(\mathcal{D})$ has two generators $\mathbf{P}_{1}, \mathbf{P}_{2}$, involving maps between two pairs of circles in the dual Descartes configuration $\mathcal{D}^{\perp}$, specified by the ordering as $A, a, B, b$, say. It takes the exterior of circle $A$ to the interior of circle $a$ and the exterior of circle $B$ to the interior of circle $b$.

\footnotetext{
${ }^{5}$ We have permuted the first and last row and column of Wilker's result because he uses the Lorentz form $w^{2}+x^{2}+y^{2}-z^{2}$.
} 


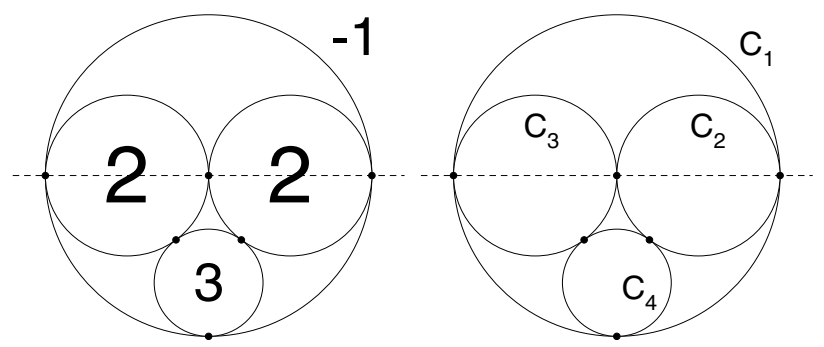

(a)

(b)

Fig. 10. Special Descartes configuration $\mathcal{D}_{0}$.

We treat the case they picture [33, Figure 7.3], where the Schottky limit set is $\Lambda\left(\mathcal{D}_{0}\right)$, for the (ordered) Descartes configuration $\mathcal{D}_{0}$ having coordinates

$$
\mathbf{W}_{\mathcal{D}_{0}}=\left[\begin{array}{rrrr}
1 & -1 & 0 & 0 \\
0 & 2 & 1 & 0 \\
0 & 2 & -1 & 0 \\
1 & 3 & 0 & -2
\end{array}\right] .
$$

This configuration is pictured in Fig. 10, with part (a) indicating curvatures and part (b) shows the ordering of circles, used later in labeling Möbius group generators $\mathfrak{s}_{i}$.

The Schottky group operations are associated to the dual Descartes configuration $\mathcal{D}_{0}^{\perp}$, which has augmented curvature-center coordinates

$$
\mathbf{W}_{\mathcal{D}_{0}^{\perp}}=\left[\begin{array}{rrrr}
0 & 0 & 0 & 1 \\
1 & 1 & -1 & -1 \\
1 & 1 & 1 & 1 \\
0 & 4 & 0 & -1
\end{array}\right] .
$$

It is pictured in Fig. 11, with (a) indicating curvatures and (b) the labeling of circles used in the Schottky group.

In Fig. 11 the Schottky group generator $\mathbf{P}_{1}$ maps the exterior of circle $A$ to the interior of circle $a$, and $\mathbf{P}_{2}$ maps the exterior of circle $B$ to the interior of circle $b$. We let $\Gamma_{S}(\mathcal{D})$ denote the Schottky group they generate. The limit set $\Lambda_{\Gamma_{S}(\mathcal{D})}$ of this Schottky group is the closed set in $\mathbb{P}^{1}(\mathbb{C})$ obtained by removing the interiors of all circles in the Apollonian

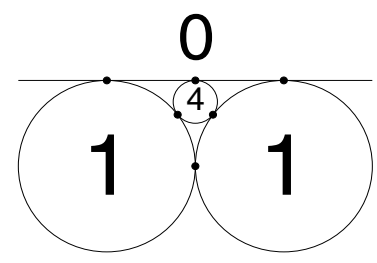

(a)

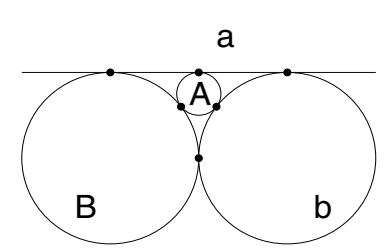

(b)

Fig. 11. Dual Descartes configuration $\mathcal{D}_{0}^{\perp}$. 
packing associated to $\mathcal{D}$. This is exactly the residual set of the Apollonian packing, see Theorem 4.2 for its properties. It is a fractal set of Hausdorff dimension about 1.3.

The precise result is as follows.

Proposition B.1. The Schottky group $\Gamma=\left\langle\mathbf{P}_{1}, \mathbf{P}_{2}\right\rangle$ given by the parabolic generators

$$
\mathbf{P}_{1}=\left[\begin{array}{cc}
1-i & 1 \\
1 & 1+i
\end{array}\right] \text { and } \mathbf{P}_{2}=\left[\begin{array}{cc}
1 & 0 \\
-2 i & 1
\end{array}\right]
$$

has a limit set $\Lambda_{\Gamma}$ which is the complement of the interiors of the circles in the Apollonian packing $\mathcal{P}_{\mathcal{D}_{0}}$ associated to the Descartes configuration $\mathcal{D}_{0}$ above.

This limit set, "the glowing gasket," is illustrated in Figure 7.3 in Chapter 7 in [33].

The Schottky group $\Gamma_{S}\left(\mathcal{D}_{0}\right)$ is very special; it admits no deformations other than conjugacy, and under conjugacy $\mathfrak{g} \Gamma_{S}\left(\mathcal{D}_{0}\right) \mathfrak{g}^{-1}=\Gamma_{S}(\mathcal{D})$ with $\mathcal{D}=\mathfrak{g}\left(\mathcal{D}_{0}\right)$. Recall that a matrix $\mathbf{M} \in \operatorname{PSL}(2, \mathbb{C})$ is parabolic if Trace $(\mathbf{M})= \pm 2$; its action on the Riemann sphere has a single fixed point. Here $\mathbf{P}_{1}, \mathbf{P}_{2}$ are parabolic, as is

$$
\left[\mathbf{P}_{1}, \mathbf{P}_{2}\right]:=\mathbf{P}_{1} \mathbf{P}_{2} \mathbf{P}_{1}^{-1} \mathbf{P}_{2}^{-1}=\left[\begin{array}{cc}
-1-2 i & 2 i \\
-2 i & -1+2 i
\end{array}\right] .
$$

The Schottky group $\Gamma_{S}\left(\mathcal{D}_{0}\right)$ is characterized up to conjugacy by the property that it is generated by two elements $\mathbf{P}_{1}, \mathbf{P}_{2}$ which are parabolic, such that their commutator $\left[\mathbf{P}_{1}, \mathbf{P}_{2}\right]=\mathbf{P}_{1} \mathbf{P}_{2} \mathbf{P}_{1}^{-1} \mathbf{P}_{2}^{-1}$ is also parabolic [33, pp. 207-208].

Now consider the group $G_{\mathcal{A}}\left(\mathcal{D}_{0}\right)=\left\langle\mathfrak{s}_{1}, \mathfrak{s}_{2}, \mathfrak{s}_{3}, \mathfrak{s}_{4}\right\rangle$ of Möbius transformations corresponding to the Apollonian group for the Descartes configuration $\mathcal{D}_{0}$, see (4.2). Here $\mathbf{s}_{i}$ is inversion in the $i$ th circle of the dual Descartes configuration $\mathcal{D}^{\perp}$, which is the circle passing through the three intersection points in $\mathcal{D}$ not touching $C_{i}$. The group $G_{\mathcal{A}}\left(\mathcal{D}_{0}\right)$ lifts to a group $\Gamma_{A}\left(\mathcal{D}_{0}\right)$ of isometries of $\mathbb{H}^{3}$. We describe $G_{\mathcal{A}}\left(\mathcal{D}_{0}\right)$ for the case that $\mathcal{D}_{0}$ is the Descartes configuration in Fig. 10 with circles numbered as in Fig. 10(b); all other $G_{\mathcal{A}}(\mathcal{D})$ are related to it by conjugacy in the Möbius group. An inversion in a circle $C$ of radius $r$ centered at $\mathbf{z}_{0}$ is the anti-holomorphic map

$$
\mathfrak{j}_{C}(z)=z_{0}+\frac{r^{2}}{\bar{z}-\overline{z_{0}}}=\frac{z_{0} \bar{z}+r^{2}-\left|z_{0}\right|^{2}}{\bar{z}-\bar{z}_{0}} .
$$

We can write the group generators as $\mathfrak{s}_{j}=\mathfrak{p}_{j} \circ \mathfrak{c}$, where $\mathfrak{p}_{j} \in \operatorname{PSL}(2, \mathbb{C})$ is holomorphic and $\mathfrak{c}$ denotes complex conjugation, with

$$
\mathfrak{p}_{1}=\left[\begin{array}{cc}
1-i & -i \\
i & 1+i
\end{array}\right], \quad \mathfrak{p}_{2}=\left[\begin{array}{cc}
1+i & -i \\
i & 1-i
\end{array}\right], \quad \mathfrak{p}_{3}=\left[\begin{array}{cc}
1 & 0 \\
4 i & 1
\end{array}\right], \quad \mathfrak{p}_{4}=\left[\begin{array}{ll}
1 & 0 \\
0 & 1
\end{array}\right] .
$$

Since $\mathfrak{p}_{4}=\mathbf{I}$, the subgroup of holomorphic elements of $G_{\mathcal{A}}(\mathcal{D})$ is

$$
\begin{aligned}
G_{\mathcal{A}}^{2}(\mathcal{D}) & =\left\langle\mathfrak{p}_{1} \overline{\mathfrak{p}}_{2}, \mathfrak{p}_{1} \overline{\mathfrak{p}_{3}}, \mathfrak{p}_{1} \overline{\mathfrak{p}}_{4}, \mathfrak{p}_{2} \overline{\mathfrak{p}_{3}}, \mathfrak{p}_{2} \overline{\mathfrak{p}_{4}}, \mathfrak{p}_{3} \overline{\mathfrak{p}_{4}}, \mathfrak{p}_{2} \overline{\mathfrak{p}_{1}}, \mathfrak{p}_{3} \overline{\mathfrak{p}_{1}}, \mathfrak{p}_{4} \overline{\mathfrak{p}}_{1}, \mathfrak{p}_{3} \overline{\mathfrak{p}_{2}}, \mathfrak{p}_{4} \overline{\mathfrak{p}_{2}}, \mathfrak{p}_{4} \overline{\mathfrak{p}_{3}}\right\rangle \\
& =\left\langle\mathfrak{p}_{1}, \mathfrak{p}_{2}, \mathfrak{p}_{3}\right\rangle,
\end{aligned}
$$

where we used the fact that $\overline{\mathfrak{p}_{j}}=\mathfrak{p}_{j}^{-1}$. 
There are clearly relations between the Schottky group $\Gamma_{S}\left(\mathcal{D}_{0}\right)$ and the group $G_{\mathcal{A}}^{2}\left(\mathcal{D}_{0}\right)$ of holomorphic elements of $G_{\mathcal{A}}(\mathcal{D})$. Indeed, the group $G_{\mathcal{A}}^{2}\left(\mathcal{D}_{0}\right)$ is generated by three parabolic elements $\mathfrak{p}_{1}, \mathfrak{p}_{2}, \mathfrak{p}_{3}$, and these are related to the three parabolic elements $P_{1}, P_{2},\left[P_{1}, P_{2}\right]$ in $\Gamma_{S}(\mathcal{D})$ by the relations

$$
\mathfrak{p}_{3}=\left(P_{2}\right)^{-2}, \quad \mathfrak{p}_{1}^{-1} \mathfrak{p}_{2}=-\left(P_{1}\right)^{-2}, \quad \mathfrak{p}_{2}^{2}=-\left[P_{1}, P_{2}\right] .
$$

However, these groups are not exactly the same. It remains to determine a more precise relation between them, explaining how they give rise to the same Apollonian packing. Note that both these groups lifted to discrete isometry groups of $\mathbb{H}^{3}$ have fundamental domains in $\mathbb{H}^{3}$ of infinite volume.

\section{Bibliography}

1. D. Aharonov and K. Stephenson, Geometric sequences of discs in the Apollonian packing, Algebra $i$ Analiz 9(3) (1997), 104-140. [English version: St. Petersburg Math. J. 9 (1998), 509-545.]

2. P. Bahls, Relative hyperbolicity and right-angled Coxeter groups, Eprint: arXiv: math.GR/041280 21 Jan 2004.

3. A. F. Beardon, The Geometry of Discrete Groups, Springer-Verlag, New York 1983.

4. M. Berger, Geometry II, Springer-Verlag, Berlin 1987.

5. D. W. Boyd, The osculatory packing of a three-dimensional sphere, Canad. J. Math. 25 (1973), 303-322.

6. D. W. Boyd, The residual set dimension of the Apollonian packing, Mathematika 20 (1973), 170-174.

7. D. W. Boyd, Improved bounds for the disk-packing constant, Aequationes Math. 9 (1973), 99-106.

8. D. W. Boyd, A new class of infinite sphere packings, Pacific J. Math. 50 (1974), 383-398.

9. D. W. Boyd, The sequence of radii of the Apollonian packing, Math. Comp. 39 (1982), 249-254.

10. R. W. Brooks, The spectral geometry of the Apollonian packing, Comm. Pure. Appl. Math. 38 (1985), 359-366.

11. J. W. S. Cassels, Rational Quadratic Forms, Academic Press, New York, 1978.

12. J. H. Conway, with F. Fung, The Sensual (Quadratic) Form, Carus Monograph No. 26, Mathematical Assocication of America, Washington, DC, 1997.

13. H. S. M. Coxeter, The problem of Apollonius, Amer. Math. Monthly 75 (1968), 5-15.

14. H. S. M. Coxeter, Loxodromic sequences of tangent spheres, Aequationes Math. 1 (1968), 104-121.

15. H. S. M. Coxeter, Introduction to Geometry, 2nd ed., Wiley, New York, 1969.

16. H. S. M. Coxeter, Numerical distances among the spheres in a loxodromic sequence, Math. Intelligencer 19 (1997), 41-47.

17. R. Descartes. Oeuvres de Descartes, Correspondence IV (C. Adam and P. Tannery, Eds.), Leopold Cerf, Paris, 1901.

18. K. J. Falconer, The Geometry of Fractal Sets, Cambridge Tracts in Mathematics, vol. 85, Cambridge University Press, Cambridge, 1985.

19. T. Gossett, The Kiss Precise, Nature 139(1937), 62.

20. T. Gossett, The Hexlet, Nature 139 (1937), 251.

21. R. L. Graham, J. C. Lagarias, C. L. Mallows, A. Wilks and C. Yan, Apollonian circle packings: number theory, J. Number Theory 100 (2003), 1-45. [Eprint: arXiv math. NT/ 009113]

22. R. L. Graham, J. C. Lagarias, C. L. Mallows, A. Wilks and C. Yan, Apollonian circle packings: geometry and group theory. II. Super-Apollonian group and integral packings, Discrete Comput. Geom. DOI: 10.1007/s00454-005-1195x. [Eprint: arXiv math.MG/0010302]

23. R. L. Graham, J. C. Lagarias, C. L. Mallows, A. Wilks and C. Yan, Apollonian circle packings: geometry and group theory. III. Higher dimensions, Discrete Comput. Geom. DOI: 10.1007/s00454-1197-8. [eprint:arXiv math.MG/0010324]

24. T. E. Heath, A History of Greek Mathematics, Volume II. From Aristarchus to Diophantus, Dover, New York, 1981. (Original: Clarendon Press, Oxford, 1921.)

25. K. E. Hirst, The Apollonian packing of circles, J. London Math. Soc. 42 (1967), 281-291. 
26. E. Kasner and F. Supnick, The Apollonian packing of circles, Proc. Natl. Acad. Sci. USA 29 (1943), 378-384.

27. R. Lachlan, On systems of circles and spheres, Philos. Trans. Roy. Soc. London Ser. A 177 (1886), 481-625.

28. J. C. Lagarias, C. L. Mallows and A. Wilks, Beyond the Descartes circle theorem, Amer. Math. Monthly 109 (2002), 338-361. [Eprint: arXiv math.MG/0101066]

29. D. G. Larman, On the exponent of convergence of a packing of spheres, Mathematika 13 (1966), 57-59.

30. B. B. Mandelbrot, The Fractal Geometry of Nature, Freeman, New York, 1983.

31. G. Maxwell, Sphere packings and hyperbolic reflection groups, J. Algebra 79 (1982), 78-97.

32. Z. A. Melzak, Infinite packings of disks, Canad. J. Math. 18 (1966), 838-853.

33. D. Mumford, C. Series and D. Wright, Indra's Pearls: The Vision of Felix Klein, Cambridge University Press, Cambridge, 2002.

34. S. Northshield, On Apollonian circle packings, Preprint.

35. D. Pedoe, On a theorem in geometry, Amer. Math. Monthly 74 (1967), 627-640.

36. B. Rodin and D. Sullivan, The convergence of circle packings to the Riemann mapping, J. Differential Geom. 26 (1987), 349-360.

37. F. Soddy, The Kiss Precise, Nature 137 (1936), 1021.

38. F. Soddy, The bowl of integers and the Hexlet, Nature 139 (1937), 77-79.

39. B. Söderberg, Apollonian tiling, the Lorentz group, and regular trees, Phys. Rev. A 46(4) (1992), 18591866.

40. P. B. Thomas and D. Dhar, The Hausdorff dimension of the Apollonian packing of circles, J. Phys. A 27 (1994), 2257-2268.

41. C. Tricot, A new proof for the residual set dimension of the Apollonian packing, Math. Proc. Cambridge Philos. Soc. 96 (1984), 413-423.

42. J. B. Wilker, Inversive Geometry, in The Geometric Vein (C. Davis, B. Grünbaum, F. A. Sherk, Eds.), Springer-Verlag, New York, 1981, pp. 379-442.

Received July 31, 2000, and in revised form March 11, 2005. Online publication September 30, 2005. 